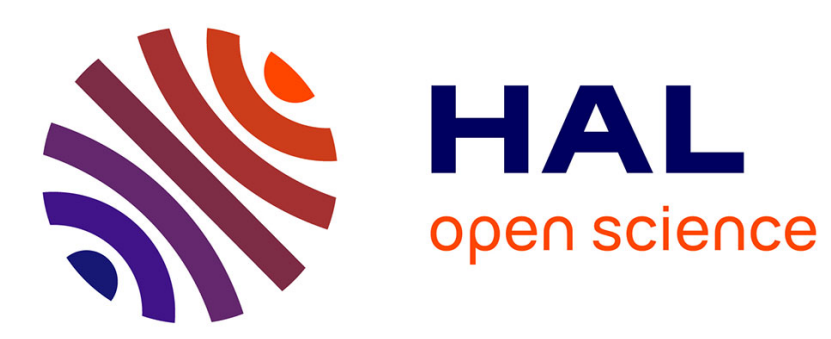

\title{
Stochastic control for mean-field Stochastic Partial Differential Equations with jumps
}

Roxana Dumitrescu, Bernt Øksendal, Agnès Sulem

\section{To cite this version:}

Roxana Dumitrescu, Bernt Øksendal, Agnès Sulem. Stochastic control for mean-field Stochastic Partial Differential Equations with jumps. Journal of Optimization Theory and Applications, 2018, pp 559-584. 10.1007/s10957-018-1243-3 . hal-01527225

\section{HAL Id: hal-01527225 \\ https://hal.inria.fr/hal-01527225}

Submitted on 24 May 2017

HAL is a multi-disciplinary open access archive for the deposit and dissemination of scientific research documents, whether they are published or not. The documents may come from teaching and research institutions in France or abroad, or from public or private research centers.
L'archive ouverte pluridisciplinaire HAL, est destinée au dépôt et à la diffusion de documents scientifiques de niveau recherche, publiés ou non, émanant des établissements d'enseignement et de recherche français ou étrangers, des laboratoires publics ou privés. 


\title{
Stochastic control of mean-field SPDEs with jumps
}

\author{
Roxana Dumitrescu* ${ }^{*}$ Bernt $\varnothing_{\text {ksendal }}^{\dagger} \quad$ Agnès Sulem ${ }^{\ddagger}$
}

May 24, 2017

\begin{abstract}
We study the problem of optimal control for mean-field stochastic partial differential equations (stochastic evolution equations) driven by a Brownian motion and an independent Poisson random measure, in the case of partial information control. One important novelty of our problem is represented by the introduction of general meanfield operators, acting on both the controlled state process and the control process. We first formulate a sufficient and a necessary maximum principle for this type of control. We then prove existence and uniqueness of the solution of such general forward and backward mean-field stochastic partial differential equations. We finally apply our results to find the explicit optimal control for an optimal harvesting problem.
\end{abstract}

Keywords: Mean-field stochastic partial differential equation (MFSPDE); optimal control; mean-field backward stochastic partial differential equation (MFBSPDE); stochastic maximum principles.

\section{Introduction}

\subsection{A motivating example}

As a motivation for the problem studied in this paper, we consider the following optimal harvesting problem: Suppose we model the density $Y(t, x)$ of a fish population in a lake

\footnotetext{
*Department of Mathematics, King's College London, United Kingdom, email: roxana.dumitrescu@kcl.ac.uk

$\dagger^{\dagger}$ Department of Mathematics, University of Oslo, P.O. Box 1053 Blindern, N-0316 Oslo, Norway, email: oksendal@math.uio.no

¥INRIA Paris, Equipe-projet MathRisk, 3 rue Simone Iff, CS 42112, 75589 Paris Cedex 12, France, email: agnes.sulem@inria.fr
} 
$D$ at time $t$ and at point $x \in D$ by an equation of the form:

$$
\begin{aligned}
d Y(t, x)= & \mathbf{E}[Y(t, x)] b(t, x) d t+\frac{1}{2} \sum_{i=1}^{d} \frac{\partial^{2}}{\partial^{2} x_{i}} Y(t, x) d t+Y(t, x) \sigma(t, x) d W_{t} \\
& +Y(t, x) \int_{\mathbf{R}^{*}} \theta(t, x, e) \tilde{N}(d t, d e) . \\
Y(0, x)= & y_{0}(x), x \in D
\end{aligned}
$$

where $D$ is a bounded domain in $\mathbf{R}^{d}$ and $y_{0}(x), b(t, x), \sigma(t, x), \theta(t, x, e)$ are given bounded deterministic functions. Here $W_{t}$ is a Brownian motion and $\tilde{N}(d t, d e)=N(d t, d e)-\nu(d e) d t$ is an independent compensated Poisson random measure, respectively, on a filtered probability space $\left(\Omega, \mathcal{F}, \mathbb{F}=\left\{\mathcal{F}_{t}\right\}, P\right)$.

We may heuristically regard (1.1) as a limit as $n \rightarrow \infty$ of a large population interacting system of the form

$$
\begin{aligned}
d y^{j, n}(t, x) & =\left[\frac{1}{n} \sum_{l=1}^{n} y^{l, n}(t, x)\right] b(t, x) d t+\frac{1}{2} \sum_{i=1}^{d} \frac{\partial^{2}}{\partial^{2} x_{i}} y^{j, n}(t, x) d t+y^{j, n}(t, x) \sigma(t, x) d W_{t} \\
& +y^{j, n}(t, x) \int_{\mathbf{R}^{*}} \theta(t, x, e) \tilde{N}(d t, d e), j=1,2, \ldots, n \\
y^{j, n}(t, x)(0, x) & =y_{0}(x)
\end{aligned}
$$

where we have divided the whole lake into a grid of size $n$ and $y^{j, n}(t, x)$ represents the density in box $j$ of the grid. Now suppose we introduce a harvesting-rate process $u(t, x)$. The density of the corresponding population $Y(t, x)=Y^{u}(t, x)$ is thus modeled by a controlled mean-field stochastic partial differential equation with jumps of the form:

$$
\begin{array}{r}
d Y(t, x)=\mathbf{E}[Y(t, x)] b(t, x) d t+\frac{1}{2} \sum_{i=1}^{d} \frac{\partial^{2}}{\partial^{2} x_{i}} Y(t, x) d t+Y(t, x) \sigma(t, x) d W_{t} \\
+Y(t, x) \int_{\mathbf{R}^{*}} \theta(t, x, e) \tilde{N}(d t, d e)-Y(t, x) u(t, x) d t
\end{array}
$$

The performance functional is assumed to be of the form

$$
J(u)=\mathbf{E}\left[\int_{0}^{T} \int_{D} \log (Y(t, x) u(t, x)) d x d t+\int_{D} \alpha(x) Y(T, x) d x\right] .
$$

This may be regarded as the expected total logarithmic utility of the harvest up to time $T$ plus the value of the remaining population at time $T$.

The problem is thus to find $u^{*}$ such that

$$
J\left(u^{*}\right)=\sup _{u \in \mathcal{A}} J(u)
$$

where $\mathcal{A}$ represents the set of admissible controls. This process $u^{*}(t, x)$ is called an optimal harvesting rate. 
This is an example of an optimal control problem for a mean-field stochastic reactiondiffusion equation. In the next sections, we will give necessary and sufficient conditions for optimality of a control in the case of partial information, as well as results of existence and uniqueness of the solution for forward and backward mean-field stochastic partial differential equations with a general mean-field operator. Finally, we apply our results in order to solve the optimal harvesting problem presented above.

Contrary to the case of mean-field (B)SDEs and related control problems (studied in many papers, see e.g. $[1,4,5,6,7,8])$, the mean-field stochastic partial differential equations have received little attention. To the best of our knowledge, the only paper that deals with optimal control of mean-field SPDEs is [21]. Our paper extends [21] in four ways: (i) we consider a more general mean-field operator; (ii) we introduce an additional general meanfield operator which acts on the control process; (iii) we add jumps; (iv) we study the optimal control problem in the case of partial information.

The paper is organized as follows: in Section 2 we show the sufficient and necessary maximum principles and apply the results to the optimal harvesting example. In Section 3 , we investigate the existence and the uniqueness of the solution of mean-field SPDEs with jumps and general mean-field operator. In Section 4, we prove the existence and the uniqueness of the solution of mean-field backward SPDEs with jumps and general mean-field operator.

\section{Maximum principles for optimal control with partial information of general mean-field SPDEs with jumps}

\subsection{Framework and formulation of the optimal control problem}

Let $\left(\Omega, \mathcal{F}, \mathbb{F}=\left\{\mathcal{F}_{t}\right\}_{0 \leq t \leq T}, P\right)$ be a filtered probability space. Let $W$ be a one-dimensional Brownian motion. Let $\mathbf{E}:=\mathbf{R}^{*}$ and $\mathcal{B}(\mathbf{E})$ be its Borel filtration. Suppose that it is equipped with a $\sigma$-finite positive measure $\nu$, satisfying $\int_{\mathbf{E}}|e|^{2} \nu(d e)<\infty$ and let $N(d t, d e)$ be a independent Poisson random measure with compensator $\nu(d e) d t$. We denote by $\tilde{N}(d t, d e)$ its compensated process, defined as $\tilde{N}(d t, d e)=N(d t, d e)-\nu(d e) d t$. For simplicity, we consider $d=1$.

We introduce the following notation:

- $\mathbf{L}^{2}(\mathbf{P}):=$ the set of random variables $X$ such that $\mathbf{E}\left[|X|^{2}\right]<\infty$.

- $\mathbf{L}^{2}(\mathbf{R}):=$ the set of measurable functions $k:(\mathbf{R}, \mathcal{B}(\mathbf{R})) \rightarrow(\mathbf{R}, \mathcal{B}(\mathbf{R}))$ with $\int_{\mathbf{R}} k^{2}(x) d x<$ $\infty$.

- $\mathbf{H}^{2}:=$ the set of real-valued predictable processes $Z(t, x)$ with $\mathbf{E}\left[\int_{0}^{T} \int_{D} Z^{2}(t, x) d x d t\right]<$ $\infty$, where $D$ a bounded domain in $\mathbf{R}$. 
- $\mathbf{L}_{\nu}^{2}:=$ the set of measurable functions $l:(\mathbf{E}, \mathcal{B}(\mathbf{E})) \rightarrow(\mathbf{R}, \mathcal{B}(\mathbf{R}))$ such that $\|l\|_{\mathbf{L}_{\nu}^{2}}^{2}:=$ $\int_{\mathbf{E}} l^{2}(e) \nu(d e)<\infty$. The set $\mathbf{L}_{\nu}^{2}$ is a Hilbert space equipped with the scalar product $<l, l^{\prime}>_{\nu}:=\int_{\mathbf{E}} l(e) l^{\prime}(e) \nu(d e)$ for all $l, l^{\prime} \in \mathbf{L}_{\nu}^{2} \times \mathbf{L}_{\nu}^{2}$.

- $\mathbf{H}_{\nu}^{2}$ : the set of predictable real-valued processes $k(t, x, \cdot)$ with $\mathbf{E}\left[\int_{0}^{T} \int_{D}\|k(t, x, \cdot)\|_{\mathbf{L}_{\nu}^{2}}\right]<$ $\infty$.

Assume that we are given a subfiltration

$$
\mathcal{E}_{t} \subseteq \mathcal{F}_{t} ; \quad t \in[0, T]
$$

representing the information available to the controller at time $t$. For example, we could have

$$
\mathcal{E}_{t}=\mathcal{F}_{(t-\delta)^{+}} \quad(\delta>0 \text { constant })
$$

meaning that the controller gets a delayed information flow compared to $\mathcal{F}_{t}$.

Consider a controlled mean-field stochastic partial differential equation $Y(t, x)=Y^{u}(t, x)$ at $(t, x)$ of the following form

$$
\begin{aligned}
& d Y(t, x)=[L Y(t, x)+b(t, x, Y(t, x), \mathbf{F}(Y(t, x)), u(t, x), \mathbf{G}(u(t, x)))] d t \\
& \quad+\sigma(t, x, Y(t, x), \mathbf{F}(Y(t, x)), u(t, x), \mathbf{G}(u(t, x))) d W_{t} \\
& \quad+\int_{\mathbf{E}} \theta(t, x, Y(t, x), \mathbf{F}(Y(t, x)), u(t, x), \mathbf{G}(u(t, x)), e) \tilde{N}(d t, d e) ; \quad(t, x) \in(0, T) \times D .
\end{aligned}
$$

with boundary conditions

$$
\begin{array}{r}
Y(0, x)=\xi(x) ; \quad x \in D \\
Y(t, x)=\eta(t, x) ; \quad(t, x) \in(0, T) \times \partial D .
\end{array}
$$

We interpret $Y$ as a weak (variational) solution to (2.6), in the sense that for $\phi \in C_{0}^{\infty}(D)$,

$$
\begin{aligned}
<Y_{t}, \phi>_{\mathbf{L}^{2}(D)} & =<y_{0}, \phi>_{\mathbf{L}^{2}(D)}+\int_{0}^{t}<Y_{s}, L^{*} \phi>d s+\int_{0}^{t}<b\left(s, Y_{s}\right), \phi>_{\mathbf{L}^{2}(D)} d s+ \\
\int_{0}^{t} & <\sigma\left(s, Y_{s}\right), \phi>_{\mathbf{L}^{2}(D)} d W_{s}+\int_{0}^{t} \int_{\mathbf{E}}<\theta\left(s, Y_{s}, e\right), \phi>_{\mathbf{L}^{2}(D)} \tilde{N}(d s, d e),
\end{aligned}
$$

where $L^{*}$ corresponds to the adjoint operator of $L$ and $<\cdot, \cdot>$ represents the duality product between $W^{1,2}(D)$ and $W^{1,2}(D)^{*}$, with $W^{1,2}(D)$ the Sobolev space of order 1. Existence and the uniqueness of the solution are proved in Section 3.

Under this framework the Itô formula can be applied to such SPDEs. See e.g. Pardoux [16], Prévot and Rockner [18].

Here, $d Y(t, x)=d_{t} Y(t, x)$ is the differential with respect to $t$ and $L$ is a bounded linear differential operator acting on $x$. The process $u(t, x, \omega)$ is our control process, taking values in an open set $\mathbf{A} \subset \mathbf{R}$. The functions $b:[0, T] \times \Omega \times D \times \mathbf{R}_{2} \times \mathbf{A} \times \mathbf{R} \mapsto \mathbf{R} ;(t, \omega, x, y, \bar{y}, u, \bar{u}) \mapsto$ $b(t, \omega, x, y, \bar{y}, u, \bar{u}), \sigma:[0, T] \times \Omega \times D \times \mathbf{R}_{2} \times \mathbf{A} \times \mathbf{R} \mapsto \mathbf{R} ;(t, \omega, x, y, \bar{y}, u, \bar{u}) \mapsto \sigma(t, \omega, x, y, \bar{y}, u, \bar{u})$, 
$\theta:[0, T] \times \Omega \times D \times \mathbf{R}_{2} \times \mathbf{A} \times \mathbf{R} \times \mathbf{E} \mapsto \mathbf{R} ;(t, \omega, x, y, \bar{y}, u, \bar{u}, e) \mapsto \theta(t, \omega, x, y, \bar{y}, u, \bar{u}, e)$ are predictable maps. We assume that $b, \sigma, \theta$ are $C_{b}^{1}$ and have linear growth with respect to $y, \bar{y}, u, \bar{u}$. We denote by $\mathcal{A}_{\mathcal{E}}$ a given family of admissible controls, contained in the set of $\mathcal{E}_{t}$-predictable stochastic processes $u(t, x) \in \mathbf{A}$ satisfying $\mathbf{E}\left[\int_{0}^{T} \int_{D} u^{2}(t, x) d x d t\right]<\infty$ and such that (2.6)-(2.7)-(2.8) has a unique càdlàg solution $Y(t, x)$.

In the above equation, $\mathbf{F}, \mathbf{G}: \mathbf{L}^{2}(\mathbf{P}) \mapsto \mathbf{R}$ are Fréchet differentiable operators. One important example is represented by the expectation operator $\mathbf{E}[\cdot]$.

Let $f:[0, T] \times \Omega \times D \times \mathbf{R}_{2} \times \mathbf{A} \times \mathbf{R} \mapsto \mathbf{R}$ and $g: \Omega \times D \times \mathbf{R}_{2} \mapsto \mathbf{R}$ be a given profit rate function and bequest rate function, respectively. Moreover, we suppose that

$$
\begin{array}{r}
\mathbf{E}\left[\int_{0}^{T}\left(\int_{D}|f(t, x, Y(t, x), \mathbf{F}(Y(t, x)), u(t, x), \mathbf{G}(u(t, x)))| d x\right) d t\right. \\
+\int_{D} \mid g(x, Y(T, x), \mathbf{F}(Y(t, x)) \mid d x]<\infty
\end{array}
$$

where $f(t, \omega, x, y, \bar{y}, u, \bar{u}), g(\omega, x, y, \bar{y})$ are measurable functions of class $C_{b}^{1}$ with respect to $(y, \bar{y}, u, \bar{u})$ and continuous w.r.t to $t$. $\mathbf{E}$ denotes the expectation with respect to $P$.

For each $u \in \mathcal{A}_{\mathcal{E}}$, we define the performance functional $J(u)$ by

$$
\begin{array}{r}
J(u)=\mathbf{E}\left[\int_{0}^{T}\left(\int_{D} f(t, x, Y(t, x), \mathbf{F}(Y(t, x)), u(t, x), \mathbf{G}(u(t, x))) d x\right) d t\right. \\
+\int_{D} g(x, Y(T, x), \mathbf{F}(Y(t, x)) \mid d x] .
\end{array}
$$

We aim to maximize $J(u)$ over all $u \in \mathcal{A}_{\mathcal{E}}$ and our problem is the following:

Find $u^{*} \in \mathcal{A}_{\mathcal{E}}$ such that

$$
\sup _{u \in \mathcal{A}} J(u)=J\left(u^{*}\right) .
$$

Such a process $u^{*}$ is called an optimal control (if it exists), and the number $J=J\left(u^{*}\right)$ is the value of this problem.

\subsection{Sufficient maximum principle for partial information optimal control for mean-field SPDEs with jumps}

In this section, we prove necessary and sufficient maximum principles for optimal control with partial information in the case of a process described by a mean-field stochastic partial differential equation (in short MFSPDE) driven by a Brownian motion $(W)$ and a Poisson random measure $\tilde{N}$. The drift and the diffusion coefficients as well as the performance functional depend not only on the state and the control but also on the distribution of the state process, and also on the one of the control.

Define the Hamiltonian $H:[0, T] \times D \times \mathbf{R}_{2} \times \mathbf{A} \times \mathbf{R}_{3} \times \mathbf{L}_{\nu}^{2} \mapsto \mathbf{R}$ as follows:

$$
\begin{array}{r}
H(t, x, y, \bar{y}, u, \bar{u}, p, q, \gamma)=f(t, x, y, \bar{y}, u, \bar{u})+b(t, x, y, \bar{y}, u, \bar{u}) p+\sigma(t, x, y, \bar{y}, u, \bar{u}) q \\
+\int_{\mathbf{E}} \theta(t, x, y, \bar{y}, u, \bar{u}, e) \gamma(e) d \nu(e) .
\end{array}
$$


In our case, since the state process and the cost functional are of mean-field type, it turns out that the adjoint equation will be a mean-field backward SPDE, denoted in the sequel MFBSPDEs.

We now introduce the adjoint operator of the operator $L$, denoted by $L^{*}$, which satisfies

$$
\left(L^{*} \phi, \psi\right)=(\phi, L \psi), \quad \text { for all } \phi, \psi \in C_{0}^{\infty}(\mathbf{R}),
$$

where

$$
<\phi_{1}, \phi_{2}>_{\mathbf{L}^{2}(\mathbf{R})}:=\left(\phi_{1}, \phi_{2}\right)=\int_{\mathbf{R}} \phi_{1}(x) \phi_{2}(x) d x
$$

is the inner product in $\mathbf{L}^{2}(\mathbf{R})$.

For $u \in \mathcal{A}_{\mathcal{E}}$, we consider the following mean field backward stochastic partial differential equation (the adjoint equation) in the three unknown processes $p(t, x) \in \mathbf{R}, q(t, x) \in$ $\mathbf{R}, \gamma(t, x, \cdot) \in \mathbf{L}_{\nu}^{2}$; called the adjoint processes:

$$
\begin{aligned}
& d p(t, x)=-\left[L^{*} p(t, x)+\frac{\partial H}{\partial y}(t, x, Y(t, x), \mathbf{F}(Y(t, x)), u(t, x), \mathbf{G}(u(t, x)), p(t, x), q(t, x), \gamma(t, x, \cdot))\right] d t \\
& -\mathbf{E}\left[\frac{\partial H}{\partial \bar{y}}(t, x, Y(t, x), \mathbf{F}(Y(t, x)), u(t, x), \mathbf{G}(u(t, x)), p(t, x), q(t, x), \gamma(t, x, \cdot))\right] \nabla \mathbf{F}(Y(t, x)) d t \\
& +q(t, x) d W_{t}+\int_{\mathbf{E}} \gamma(t, x, e) \tilde{N}(d t, d e) ;(t, x) \in(0, T) \times D . \\
& p(T, x)=\frac{\partial g}{\partial y}(x, Y(T, x), \mathbf{F}(Y(T, x)))+\mathbf{E}\left[\frac{\partial g}{\partial \bar{y}}(x, Y(T, x), \mathbf{F}(Y(T, x))] \nabla \mathbf{F}(Y(T, x)) ; x \in D\right.
\end{aligned}
$$

$p(t, x)=0 ; \quad(t, x) \in(0, T) \times \partial D$.

Note that (2.14) is equivalent to

$$
\begin{aligned}
d p(t, x) & =-\left[L^{*} p(t, x)+\frac{\partial f}{\partial y}(t, x, Y(t, x), \mathbf{F}(Y(t, x)), u(t, x), \mathbf{G}(u(t, x)))\right. \\
& \left.+\frac{\partial b}{\partial y}(t, x, Y(t, x), \mathbf{F}(Y(t, x)), u(t, x), \mathbf{G}(u(t, x))) p(t, x)\right] d t \\
& -\left[\frac{\partial \sigma}{\partial y}(t, x, Y(t, x), \mathbf{F}(Y(t, x)), u(t, x), \mathbf{G}(u(t, x))) q(t, x)\right. \\
& \left.+\int_{\mathbf{E}} \frac{\partial \theta}{\partial y}(t, x, Y(t, x), \mathbf{F}(Y(t, x)), u(t, x), \mathbf{G}(u(t, x)), e) \gamma(t, x, e) \nu(d e)\right] d t \\
& -\mathbf{E}\left[\frac{\partial f}{\partial \bar{y}}(t, x, Y(t, x), \mathbf{F}(Y(t, x)), u(t, x), \mathbf{G}(u(t, x)))\right.
\end{aligned}
$$




$$
\begin{aligned}
& \left.+\frac{\partial b}{\partial \bar{y}}(t, x, Y(t, x), \mathbf{F}(Y(t, x)), u(t, x), \mathbf{G}(u(t, x))) p(t, x)\right] \nabla \mathbf{F}(Y(t, x)) d t \\
& -\mathbf{E}\left[\frac{\partial \sigma}{\partial \bar{y}}(t, x, Y(t, x), \mathbf{F}(Y(t, x)), u(t, x), \mathbf{G}(u(t, x))) q(t, x)\right] \nabla \mathbf{F}(Y(t, x)) d t \\
& -\mathbf{E}\left[\int_{\mathbf{E}} \frac{\partial \theta}{\partial \bar{y}}(t, x, Y(t, x), \mathbf{F}(Y(t, x)), u(t, x), \mathbf{G}(u(t, x)), e) \gamma(t, x, e) \nu(d e)\right] \nabla \mathbf{F}(Y(t, x)) d t \\
& +q(t, x) d W_{t}+\int_{\mathbf{E}} \gamma(t, x, e) \tilde{N}(d t, d e), x \in D .
\end{aligned}
$$

We now show the sufficient maximum principle.

Theorem 2.1 (Sufficient Maximum Principle for mean-field SPDEs with jumps) Let $\hat{u} \in \mathcal{A}_{\mathcal{E}}$ with corresponding solution $\hat{Y}(t, x)$ and suppose that $\hat{p}(t, x), \hat{q}(t, x)$ and $\hat{\gamma}(t, x, \cdot)$ is a solution of the adjoint MFBSPDE (2.14)-(2.15)-(2.16). Assume the following hold:

(i) The maps $Y \mapsto g(x, Y, \boldsymbol{F}(Y))$ and

$$
(Y, u) \mapsto H(Y, u):=H(t, x, Y, \boldsymbol{F}(Y), u, \boldsymbol{G}(u), \hat{p}(t, x), \hat{q}(t, x), \hat{\gamma}(t, x, \cdot))
$$

are concave functions with respect to $Y$ and $(Y, u)$, respectively, for all $(t, x) \in[0, T] \times$ D.

(ii) (The maximum condition)

$$
\begin{array}{r}
\mathbf{E}\left[H(t, x, \hat{Y}(t, x), \boldsymbol{F}(\hat{Y}(t, x)), \hat{u}(t, x), \boldsymbol{G}(\hat{u}(t, x)), \hat{p}(t, x), \hat{q}(t, x), \hat{\gamma}(t, x, \cdot)) \mid \mathcal{E}_{t}\right]= \\
e s s \sup _{v \in \mathcal{A}_{\mathcal{E}}} \mathbf{E}\left[H(t, x, \hat{Y}(t, x), \boldsymbol{F}(\hat{Y}(t, x)), v(t, x), \boldsymbol{G}(v(t, x)), \hat{p}(t, x), \hat{q}(t, x), \hat{\gamma}(t, x, \cdot)) \mid \mathcal{E}_{t}\right] \quad \text { a.s. }
\end{array}
$$

for all $t \in[0, T]$ and $x \in D$.

Then $\hat{u}(t)$ is an optimal control for the random jump field control problem (2.11).

Proof. Define a sequence of stopping times $\tau_{n} ; n=1,2, \ldots$ as follows:

$\tau_{n}:=\inf \left\{t>0 ; \max \left\{\|\hat{p}(t)\|_{\mathbf{L}^{2}(D)},\|\hat{q}(t)\|_{\mathbf{L}^{2}(D)},\|\hat{\gamma}(t)\|_{\mathbf{L}^{2}(D \times \mathbf{E})},\|\sigma(t)-\hat{\sigma}(t)\|_{\mathbf{L}^{2}(D)},\|\theta(t)-\hat{\theta}(t)\|_{\mathbf{L}^{2}(D \times \mathbf{E})}\right.\right.$,

$$
\left.\|Y(t)-\hat{Y}(t)\|_{\mathbf{L}^{2}(D)} \geq n\right\} \wedge T .
$$

Then $\tau_{n} \rightarrow T$ as $n \rightarrow \infty$ and

$$
\begin{aligned}
\mathbf{E} & {\left[\int_{0}^{\tau_{n}}\left(\int_{D} \hat{p}(t, x)(\sigma(t, x)-\hat{\sigma}(t, x)) d x\right) d W_{t}+\int_{0}^{\tau_{n}} \int_{\mathbf{E}}\left(\int_{D}(\theta(t, x, e)-\hat{\theta}(t, x, e)) d x\right) \tilde{N}(d t, d e)\right] } \\
= & \mathbf{E}\left[\int_{0}^{\tau_{n}}\left(\int_{D}(Y(t, x)-\hat{Y}(t, x)) \hat{q}(t, x) d x\right) d W_{t}\right. \\
& \left.+\int_{0}^{\tau_{n}} \int_{\mathbf{E}}\left(\int_{D}(Y(t, x)-\hat{Y}(t, x)) \hat{\gamma}(t, x, e) d x\right) \tilde{N}(d t, d e)\right]=0 \text { for all } n .
\end{aligned}
$$


Let us fix $u \in \mathcal{A}_{\mathcal{E}}$ and let $Y(t, x)=Y^{u}(t, x)$ be the associated solution of (2.6). Define:

$$
\left\{\begin{array}{l}
\hat{f}:=f(t, x, \hat{Y}(t, x), \mathbf{F}(\hat{Y}(t, x)), \hat{u}(t, x), \mathbf{G}(\hat{u}(t, x))) ; \quad f:=f(t, x, Y(t, x), \mathbf{F}(Y(t, x)), u(t, x), \mathbf{G}(u(t, x))) \\
\hat{g}:=g(x, \hat{Y}(T, x), \mathbf{F}(\hat{Y}(T, x))) ; g:=g(x, Y(T, x), \mathbf{F}(Y(T, x))) ;
\end{array}\right.
$$

and

$$
\left\{\begin{array}{l}
\hat{b}:=b(t, x, \hat{Y}(t, x), \mathbf{F}(\hat{Y}(t, x)), \hat{u}(t, x), \mathbf{G}(\hat{u}(t, x))) ; \quad b:=b(t, x, Y(t, x), \mathbf{F}(Y(t, x)), u(t, x), \mathbf{G}(u(t, x))) ; \\
\hat{\sigma}:=\sigma(t, x, \hat{Y}(t, x), \mathbf{F}(\hat{Y}(t, x)), \hat{u}(t, x), \mathbf{G}(\hat{u}(t, x))) ; \sigma:=\sigma(t, x, Y(t, x), \mathbf{F}(Y(t, x)), u(t, x), \mathbf{G}(u(t, x))) \\
\hat{\theta}:=\theta(t, x, \hat{Y}(t, x), \mathbf{F}(\hat{Y}(t, x)), \hat{u}(t, x), \mathbf{G}(\hat{u}(t, x)), e) ; \\
\theta:=\theta(t, x, Y(t, x), \mathbf{F}(Y(t, x)), u(t, x), \mathbf{G}(u(t, x)), e) .
\end{array}\right.
$$

We also set

$$
\left\{\begin{array}{l}
\hat{H}:=H(t, x, \hat{Y}(t, x), \mathbf{F}(\hat{Y}(t, x)), \hat{u}(t, x), \mathbf{G}(\hat{u}(t, x)), \hat{p}(t, x), \hat{q}(t, x), \hat{\gamma}(t, x, \cdot)) ; \\
H:=H(t, x, Y(t, x), \mathbf{F}(Y(t, x)), u(t, x), \mathbf{G}(u(t, x)), \hat{p}(t, x), \hat{q}(t, x), \hat{\gamma}(t, x, \cdot)) .
\end{array}\right.
$$

Using the above definitions and the definition of the performance functional $J$, we get that:

$$
J(u)-J(\hat{u})=\mathcal{J}_{1}+\mathcal{J}_{2},
$$

where $\mathcal{J}_{1}:=\mathbf{E}\left[\int_{0}^{T} \int_{D}(f-\hat{f}) d x d t\right]$ and $\mathcal{J}_{2}:=\mathbf{E}\left[\int_{D}(g-\hat{g}) d x\right]$.

Now, let us notice the following relations:

$$
\left\{\begin{array}{l}
\hat{f}=\hat{H}-\hat{b} \hat{p}(t, x)-\hat{\sigma} \hat{q}(t, x)-\int_{\mathbf{E}} \hat{\theta} \hat{\gamma}(t, x, e) \nu(d e) \\
\hat{f}=H-b \hat{p}(t, x)-\sigma \hat{q}(t, x)-\int_{\mathbf{E}} \theta \hat{\gamma}(t, x, e) \nu(d e)
\end{array}\right.
$$

which imply

$$
\mathcal{J}_{1}=\mathbf{E}\left[\int_{0}^{T} \int_{D}\left(H-\hat{H}-(b-\hat{b}) \cdot \hat{p}-(\sigma-\hat{\sigma}) \cdot \hat{q}-\int_{\mathbf{E}}(\theta-\hat{\theta}) \cdot \hat{\gamma} \nu(d e)\right)\right] .
$$

Fix $x \in D$. Since the map $Y \mapsto g(x, Y, F(Y))$ is concave for each $x \in \bar{D}$, we obtain:

$$
g-\hat{g} \leq \frac{\partial g}{\partial y}(x, \hat{Y}(T, x), \mathbf{F}(\hat{Y}(T, x))) \tilde{Y}(T, x)+\frac{\partial g}{\partial \bar{y}}(x, \hat{Y}(T, x), \mathbf{F}(\hat{Y}(T, x)))<\nabla \mathbf{F}(\hat{Y}(T, x)), \tilde{Y}(T, x)>_{\mathbf{L}^{2}(\mathbf{P})},
$$

where

$$
\tilde{Y}(t, x)=Y(t, x)-\hat{Y}(t, x) .
$$

We thus obtain, by taking the expectation and applying the Itô formula for jump-diffusion 
processes,

$$
\begin{aligned}
\mathcal{J}_{2} \leq \mathbf{E}\left[\int _ { D } \left(\frac{\partial g}{\partial y}(x, \hat{Y}(T, x), \mathbf{F}(\hat{Y}(T, x))) \tilde{Y}(T, x)\right.\right. & \partial g \\
+ & \left.\left.\frac{\partial g}{\partial \bar{y}}(x, \hat{Y}(T, x), \mathbf{F}(\hat{Y}(T, x)))<\nabla \mathbf{F}(\hat{Y}(T, x)), \tilde{Y}(T, x)>_{\mathbf{L}^{2}(\mathbf{P})}\right) d x\right] \\
= & \mathbf{E}\left[\int_{D}<\hat{p}(T, x), \tilde{Y}(T, x)>d x\right] \\
= & \mathbf{E}\left[\int_{D}\left(\hat{p}(0, x) \cdot \tilde{Y}(0, x)+\int_{0}^{T}(<\tilde{Y}(t, x), d \hat{p}(t, x)>+\hat{p}(t, x) d \tilde{Y}(t, x)+(\sigma-\hat{\sigma}) \hat{q}(t, x)) d t\right) d x\right] \\
+ & \mathbf{E}\left[\int_{D}\left(\int_{0}^{T} \int_{\mathbf{E}}(\theta-\hat{\theta}) \hat{\gamma}(t, x, e) N(d t, d e)\right) d x\right] \\
= & \mathbf{E}\left[\int_{D} \int_{0}^{T} \hat{p}(t, x)(L \tilde{Y}(t, x)+(b-\hat{b}))\right. \\
+ & \left.\tilde{Y}(t, x)\left(-L^{*} \hat{p}(t, x)-\frac{\hat{\partial H}}{\partial y}-\mathbf{E}\left[\frac{\partial H}{\partial \bar{y}}\right]<\nabla \mathbf{F}(\hat{Y}(t, x)), \tilde{Y}(t, x)>_{\mathbf{L}^{2}(P)}\right) d t d x\right] \\
+ & \mathbf{E}\left[\int_{D} \int_{0}^{T}\left((\sigma-\hat{\sigma}) \hat{q}(t, x)+\int_{\mathbf{E}}(\theta-\hat{\theta}) \hat{\gamma}(t, x, e) \nu(d e)\right) d t d x\right],
\end{aligned}
$$

where

$$
\frac{\overline{\partial H}}{\partial y}:=\frac{\partial H}{\partial y}(t, x, \hat{Y}(t, x), \mathbf{F}(\hat{Y}(t, x)), \hat{u}(t, x), \mathbf{G}(\hat{u}(t, x)), \hat{p}(t, x), \hat{q}(t, x), \hat{\gamma}(t, x, \cdot))
$$

and

$$
\frac{\partial H}{\partial \bar{y}}:=\frac{\partial H}{\partial \bar{y}}(t, x, \hat{Y}(t, x), \mathbf{F}(\hat{Y}(t, x)), \hat{u}(t, x), \mathbf{G}(\hat{u}(t, x)), \hat{p}(t, x), \hat{q}(t, x), \hat{\gamma}(t, x, \cdot)) .
$$

From (2.21), (2.22) and (2.23), we derive

$$
\begin{aligned}
J(u)-J(\hat{u}) & \leq \mathbf{E}\left[\int_{0}^{T}\left(\int_{D} \hat{p}(t, x) L \tilde{Y}(t, x)-\tilde{Y}(t, x) L^{*} \hat{p}(t, x) d x\right) d t\right] \\
& +\mathbf{E}\left[\int_{D}\left(\int_{0}^{T}\left(H-\hat{H}-\frac{\overline{\partial H}}{\partial y} \cdot \tilde{Y}(t, x)-\mathbf{E}\left[\frac{\partial H}{\partial \bar{y}}\right]<\nabla \mathbf{F}(\hat{Y}(t, x)), \tilde{Y}(t, x)>_{\mathbf{L}^{2}(\mathbf{P})}\right) d t\right) d x\right]
\end{aligned}
$$

Since $\tilde{Y}(t, x)=\hat{p}(t, x)=0$ for all $(t, x) \in[0, T] \times \partial D$, we obtain by an easy extension of (2.13) using Green's formula that

$$
\int_{D} \tilde{Y}(t, x) L^{*} \hat{p}(t, x) d x=\int_{D} \hat{p}(t, x) L \tilde{Y}(t, x),
$$

for all $t \in(0, T)$. We therefore get

$$
J(u)-J(\hat{u}) \leq \mathbf{E}\left[\int_{D}\left(\int_{0}^{T}\left(H-\hat{H}-\frac{\overline{\partial H}}{\partial y} \cdot \tilde{Y}(t, x)+\mathbf{E}\left[\frac{\partial H}{\partial \bar{y}}\right]<\nabla \mathbf{F}(\hat{Y}(t, x)), \tilde{Y}(t, x)>_{\mathbf{L}^{2}(P)}\right) d t\right) d x\right]
$$


By the concavity assumption (2.18) we have

$$
\begin{aligned}
H-\hat{H} \leq & \frac{\partial H}{\partial y}(\hat{Y}, \mathbf{F}(\hat{Y}), \hat{u}, \mathbf{G}(\hat{u}))(Y-\hat{Y})+\frac{\partial H}{\partial \bar{y}}(\hat{Y}, \mathbf{F}(\hat{Y}), \hat{u}, \mathbf{G}(\hat{u}))<\nabla \mathbf{F}(\hat{Y}),(Y-\hat{Y})>_{\mathbf{L}^{2}(\mathbf{P})} \\
& +\frac{\partial H}{\partial u}(\hat{Y}, \mathbf{F}(\hat{Y}), \hat{u}, \mathbf{G}(\hat{u}))(u-\hat{u})+\frac{\partial H}{\partial \bar{u}}(\hat{Y}, \mathbf{F}(\hat{Y}), \hat{u}, \mathbf{G}(\hat{u}))<\nabla \mathbf{G}(\hat{u}),(u-\hat{u})>_{\mathbf{L}^{2}(\mathbf{P})} .
\end{aligned}
$$

Combining the two above relations we get:

$$
\begin{aligned}
J(u)-J(\hat{u}) \leq & \mathbf{E}\left[\int _ { D } \int _ { 0 } ^ { T } \left(\frac{\partial H}{\partial u}(\hat{Y}, \mathbf{F}(\hat{Y}), \hat{u}, \mathbf{G}(\hat{u}))(u-\hat{u})\right.\right. \\
& \left.\left.+\frac{\partial H}{\partial \bar{u}}(\hat{Y}, \mathbf{F}(\hat{Y}), \hat{u}, \mathbf{G}(\hat{u}))<\nabla \mathbf{G}(\hat{u}),(u-\hat{u})>_{\mathbf{L}^{2}(\mathbf{P})}\right) d t d x\right] .
\end{aligned}
$$

By the maximum condition (2.19), we obtain:

$\mathbf{E}\left[\frac{\partial H}{\partial u}(\hat{Y}, \mathbf{F}(\hat{Y}), \hat{u}, \mathbf{G}(\hat{u})) \mid \mathcal{E}_{t}\right](u-\hat{u})+\mathbf{E}\left[\frac{\partial H}{\partial \bar{u}}(\hat{Y}, \mathbf{F}(\hat{Y}), \hat{u}, \mathbf{G}(\hat{u})) \mid \mathcal{E}_{t}\right]<\nabla \mathbf{G}(\hat{u}), u-\hat{u}>_{\mathbf{L}^{2}(\mathbf{P})} \leq 0$ a.s.

for all $(t, x) \in[0, T] \times D$. From (2.24) and (2.25) we conclude that

$$
J(u) \leq J(\hat{u}) .
$$

By arbitrariness of $u$, we conclude that $\hat{u}$ is optimal.

\subsection{A necessary-type maximum principle for partial information control of mean-field SPDEs with jumps}

As in many applications the concavity condition may not hold, we prove a version of the maximum principle which does not need this assumption. Instead, we assume the following:

(A1) For all $s \in[0, T)$ and all bounded $\mathcal{E}_{s}$-measurable random variables $\theta(\omega, x)$ the control $\beta$ defined by

$$
\beta_{t}(\omega, x)=\theta(\omega, x) \chi_{(s, T]}(t) ; t \in[0, T], x \in D
$$

is in $\mathcal{A}_{\mathcal{E}}$.

(A2) For all $u, \beta \in \mathcal{U}$ where $\beta$ is bounded there exists $\delta>0$ such that the control

$$
u(t)+y \beta(t) ; t \in[0, T]
$$

belongs to $\mathcal{A}_{\mathcal{E}}$ for all $y \in(-\delta, \delta)$.

Let us give an auxiliary lemma. 
Lemma 2.2 Let $u \in \mathcal{A}_{\mathcal{E}}$ and $v \in \mathcal{A}_{\mathcal{E}}$. The derivative process

$$
\mathcal{Y}(t, x):=\lim _{z \mapsto 0^{+}} \frac{Y^{u+z \beta}(t, x)-Y^{u}(t, x)}{z}
$$

exists and belongs to $\boldsymbol{L}^{2}(d x \times d t \times d P)$. We then have that $\mathcal{Y}$ satisfies the following mean-field SPDE:

$$
\begin{aligned}
& d \mathcal{Y}(t, x)=L \mathcal{Y}(t, x)+\left(\frac{\partial b}{\partial y}\left(t, x, Y^{u}(t, x), \boldsymbol{F}\left(Y^{u}(t, x)\right), \boldsymbol{G}(u(t, x))\right) \mathcal{Y}(t, x)\right. \\
& +\frac{\partial b}{\partial \bar{y}}\left(t, x, Y^{u}(t, x), \boldsymbol{F}\left(Y^{u}(t, x)\right), u(t, x), \boldsymbol{G}(u(t, x))\right)<\nabla \boldsymbol{F}\left(Y^{u}(t, x)\right), \mathcal{Y}(t, x)>_{\mathbf{L}^{2}(\boldsymbol{P})} \\
& +\frac{\partial b}{\partial u}\left(t, x, Y^{u}(t, x), \boldsymbol{F}\left(Y^{u}(t, x)\right), u(t, x), \boldsymbol{G}(u(t, x))\right) \beta(t, x) \\
& \left.+\frac{\partial b}{\partial \bar{u}}\left(t, x, Y^{u}(t, x), \boldsymbol{F}\left(Y^{u}(t, x)\right), u(t, x), \boldsymbol{G}(u(t, x))\right)<\nabla \boldsymbol{G}(u(t, x)), \beta(t, x)>_{\mathbf{L}^{2}(\boldsymbol{P})}\right) d t \\
& +\left(\frac{\partial \sigma}{\partial y}\left(t, x, Y^{u}(t, x), \boldsymbol{F}\left(Y^{u}(t, x)\right), u(t, x), \boldsymbol{G}(u(t, x))\right) \mathcal{Y}(t, x)\right. \\
& +\frac{\partial \sigma}{\partial \bar{y}}\left(t, x, Y^{u}(t, x), \boldsymbol{F}\left(Y^{u}(t, x)\right), u(t, x), \boldsymbol{G}(u(t, x))\right)<\nabla \boldsymbol{F}\left(Y^{u}(t, x)\right), \mathcal{Y}(t, x)>_{\mathbf{L}^{2}(\boldsymbol{P})} \\
& +\frac{\partial \sigma}{\partial u}\left(t, x, Y^{u}(t, x), \boldsymbol{F}\left(Y^{u}(t, x)\right), u(t, x), \boldsymbol{G}\left(Y^{u}(t, x)\right)\right) \beta(t, x) \\
& \left.+\frac{\partial \sigma}{\partial \bar{u}}\left(t, x, Y^{u}(t, x), \boldsymbol{F}\left(Y^{u}(t, x)\right), u(t, x), \boldsymbol{G}(u(t, x))\right)<\nabla \boldsymbol{G}(u(t, x)), \beta(t, x)>_{\mathbf{L}^{2}(\boldsymbol{P})}\right) d W_{t} \\
& +\int_{\mathbf{E}}\left(\frac{\partial \theta}{\partial y}\left(t, x, Y^{u}(t, x), \boldsymbol{F}\left(Y^{u}(t, x)\right), u(t, x), \boldsymbol{G}(u(t, x)), e\right) \mathcal{Y}(t, x)\right. \\
& +\frac{\partial \theta}{\partial \bar{y}}\left(t, x, Y^{u}(t, x), \boldsymbol{F}\left(Y^{u}(t, x)\right), u(t, x), \boldsymbol{G}(u(t, x)), e\right)<\nabla \boldsymbol{F}\left(Y^{u}(t, x)\right), \mathcal{Y}(t, x)>_{\mathbf{L}^{2}(\boldsymbol{P})} \\
& +\frac{\partial \theta}{\partial u}\left(t, x, Y^{u}(t, x), \boldsymbol{F}\left(Y^{u}(t, x)\right), u(t, x), \boldsymbol{G}(u(t, x)), e\right) \beta(t, x) \\
& \left.\left.+\frac{\partial \theta}{\partial \bar{u}}\left(t, x, Y^{u}(t, x), \boldsymbol{F}\left(Y^{u}(t, x)\right), u(t, x), \boldsymbol{G}(u(t, x)), e\right) \beta(t, x)\right)<\nabla \boldsymbol{G}(u(t, x)), \beta(t, x)>_{\mathbf{L}^{2}(\boldsymbol{P})}\right) \tilde{N}(d t, d e), \\
& \mathcal{Y}(t, x)=0, \quad(t, x) \in(0, T) \times \partial D \text {; } \\
& \mathcal{Y}(0, x)=0, x \in D \text {. }
\end{aligned}
$$

Proof. The result follows by applying the mean theorem. We omit the details.

We now provide the necessary-type maximum principle for our optimal control problem for mean-field SPDEs.

Theorem 2.3 (Necessary-type maximum principle for mean-field SPDEs with jumps) Let $\hat{u} \in \mathcal{A}_{\mathcal{E}}$ with corresponding solutions (2.6)-(2.7)-(2.8) and (2.14)-(2.15)-(2.16). Assume 
that Assumptions (A1)-(A2) hold. Then the following are equivalent:

(i) $\left.\frac{d}{d y} J(\hat{u}+y \beta)\right|_{y=0}=0$ for all bounded $\beta \in \mathcal{A}_{\mathcal{E}}$.

(ii) $\mathbf{E}\left[\nabla \hat{H}(t, x) \mid \mathcal{E}_{t}\right]=0, \quad$ for all $(t, x) \in[0, T] \times D$ a.s. ,

where

$$
\begin{array}{r}
\nabla \hat{H}(t, x):=\frac{\partial H}{\partial u}(t, x, \hat{u}(t, x), \hat{Y}(t, x), \boldsymbol{F}(\hat{Y}(t, x)), \boldsymbol{G}(\hat{u}(t, x)), \hat{p}(t, x), \hat{q}(t, x), \hat{\gamma}(t, x, \cdot)) \\
+\mathbf{E}\left[\frac{\partial H}{\partial \bar{u}}(t, x, \hat{u}(t, x), \hat{Y}(t, x), \boldsymbol{F}(\hat{Y}(t, x)), \boldsymbol{G}(\hat{u}(t, x)), \hat{p}(t, x), \hat{q}(t, x), \hat{\gamma}(t, x, \cdot))\right] \nabla \boldsymbol{G}(\hat{u}(t, x)),
\end{array}
$$

for all $(t, x) \in[0, T] \times D$.

Proof. The assumptions on the coefficients together with the mean theorem and relation (2.26) yield to:

$$
\begin{aligned}
\lim _{y \rightarrow 0} & \frac{1}{y}(J(\hat{u}+y \beta)-J(\hat{u}))=\mathbf{E}\left[\int _ { 0 } ^ { T } \int _ { D } \left(\frac{\partial f}{\partial y}(t, x, \hat{Y}(t, x), \mathbf{F}(\hat{Y}(t, x)), \hat{u}(t, x), \mathbf{G}(\hat{u}(t, x))) \mathcal{Y}(t, x)\right.\right. \\
& +\frac{\partial f}{\partial \bar{y}}(t, x, \hat{Y}(t, x), \mathbf{F}(\hat{Y}(t, x)), \hat{u}(t, x), \mathbf{G}(\hat{u}(t, x)))<\nabla \mathbf{F}(\hat{Y}(t, x)), \mathcal{Y}(t, x)>_{\mathbf{L}^{2}(\mathbf{P})} \\
& \left.\left.+\frac{\partial f}{\partial u}(t, x, \hat{Y}(t, x), \mathbf{F}(\hat{Y}(t, x)), \hat{u}(t, x), \mathbf{G}(\hat{u}(t, x))) \beta(t, x)\right) d x d t\right] \\
& \left.\left.+\frac{\partial f}{\partial \bar{u}}(t, x, \hat{Y}(t, x), \mathbf{F}(\hat{Y}(t, x)), \hat{u}(t, x), \mathbf{G}(\hat{u}(t, x))) d x d t\right]<\nabla \mathbf{G}(\hat{u}(t, x)), \beta(t, x)>_{\left.\mathbf{L}^{2}(\mathbf{P})\right]}\right] \\
& +\mathbf{E}\left[\int _ { D } \left(\frac{\partial g}{\partial y}(T, x, \hat{Y}(T, x), \mathbf{F}(\hat{Y}(T, x))) \mathcal{Y}(T, x)\right.\right. \\
& \left.\left.+\frac{\partial g}{\partial \bar{y}}(T, x, \hat{Y}(T, x), \mathbf{F}(\hat{Y}(T, x)), \hat{u}(T, x))<\nabla \mathbf{F}(\hat{Y}(T, x)), \mathcal{Y}(T, x)>_{\mathbf{L}^{2}(\mathbf{P})} d x\right)\right] .
\end{aligned}
$$

The definition of the Hamiltonian $H$ implies:

$$
\begin{aligned}
& \frac{\partial f}{\partial y}(t, x, \hat{Y}(t, x), \mathbf{F}(\hat{Y}(t, x)), \hat{u}(t, x), \mathbf{G}(\hat{u}(t, x)))= \\
& =\frac{\partial \hat{H}}{\partial y}(t, x)-\frac{\partial \hat{b}}{\partial y}(t, x) \hat{p}(t, x)-\frac{\partial \hat{\sigma}}{\partial y}(t, x) \hat{q}(t, x)-\int_{\mathbf{E}} \frac{\partial \hat{\theta}}{\partial y}(t, x, e) \hat{\gamma}(t, x, e) \nu(d e) \\
& \frac{\partial f}{\partial \bar{y}}(t, x, \hat{Y}(t, x), \mathbf{F}(\hat{Y}(t, x)), \hat{u}(t, x), \mathbf{G}(\hat{u}(t, x)))= \\
& =\frac{\partial \hat{H}}{\partial \bar{y}}(t, x)-\frac{\partial \hat{b}}{\partial \bar{y}}(t, x) \hat{p}(t, x)-\frac{\partial \hat{\sigma}}{\partial \bar{y}}(t, x) \hat{q}(t, x)-\int_{\mathbf{E}} \frac{\partial \hat{\theta}}{\partial \bar{y}}(t, x, e) \hat{\gamma}(t, x, e) \nu(d e)
\end{aligned}
$$




$$
\begin{aligned}
& \frac{\partial f}{\partial u}(t, x, \hat{Y}(t, x), \mathbf{F}(\hat{Y}(t, x)), \hat{u}(t, x), \mathbf{G}(\hat{u}(t, x))) \\
& =\frac{\partial \hat{H}}{\partial u}(t, x)-\frac{\partial \hat{b}}{\partial u}(t, x) \hat{p}(t, x)-\frac{\partial \hat{\sigma}}{\partial u}(t, x) \hat{q}(t, x)-\int_{\mathbf{E}} \frac{\partial \hat{\theta}}{\partial u}(t, x, e) \hat{\gamma}(t, x, e) \nu(d e) \\
& \frac{\partial f}{\partial \bar{u}}(t, x, \hat{Y}(t, x), \mathbf{F}(\hat{Y}(t, x)), \hat{u}(t, x), \mathbf{G}(\hat{u}(t, x))) \\
& =\frac{\partial \hat{H}}{\partial \bar{u}}(t, x)-\frac{\partial \hat{b}}{\partial \bar{u}}(t, x) \hat{p}(t, x)-\frac{\partial \hat{\sigma}}{\partial \bar{u}}(t, x) \hat{q}(t, x)-\int_{\mathbf{E}} \frac{\partial \hat{\theta}}{\partial \bar{u}}(t, x, e) \hat{\gamma}(t, x, e) \nu(d e)
\end{aligned}
$$

Using (2.27), (2.28), (2.29), (2.30), (2.31) we derive:

$$
\begin{aligned}
& \lim _{y \rightarrow 0} \frac{1}{y}(J(\hat{u}+y \beta)-J(\hat{u})) \\
& =\mathbf{E}\left[\int_{0}^{T} \int_{D}\left(\frac{\partial \hat{H}}{\partial y}(t, x)-\frac{\partial \hat{b}}{\partial y}(t, x) \hat{p}(t, x)-\frac{\partial \hat{\sigma}}{\partial y}(t, x) \hat{q}(t, x)-\int_{\mathbf{E}} \frac{\partial \hat{\theta}}{\partial y}(t, x, e) \hat{\gamma}(t, x, e) \nu(d e)\right) \mathcal{Y}(t, x) d x d t\right] \\
& +\mathbf{E}\left[\int_{0}^{T} \int_{D}\left(\frac{\partial \hat{H}}{\partial \bar{y}}(t, x)-\frac{\partial \hat{b}}{\partial \bar{y}}(t, x) \hat{p}(t, x)-\frac{\partial \hat{\sigma}}{\partial \bar{y}}(t, x) \hat{q}(t, x)-\int_{\mathbf{E}} \frac{\partial \hat{\theta}}{\partial \bar{y}}(t, x, e) \hat{\gamma}(t, x, e) \nu(d e)\right)\right. \\
& \left.\quad+<\nabla \mathbf{F}(\hat{Y}(t, x)), \mathcal{Y}(t, x) \mathbf{L}^{2}(\mathbf{P}) d x d t\right] \\
& +\mathbf{E}\left[\int_{0}^{T} \int_{D}\left(\frac{\partial \hat{H}}{\partial u}(t, x)-\frac{\partial \hat{b}}{\partial u}(t, x) \hat{p}(t, x)-\frac{\partial \hat{\sigma}}{\partial u}(t, x) \hat{q}(t, x)-\int_{\mathbf{E}} \frac{\partial \hat{\theta}}{\partial u}(t, x, e) \hat{\gamma}(t, x, e) \nu(d e)\right) \beta(t, x) d x d t\right] \\
& +\mathbf{E}\left[\int_{0}^{T} \int_{D}\left(\frac{\partial \hat{H}}{\partial \bar{u}}(t, x)-\frac{\partial \hat{b}}{\partial \bar{u}}(t, x) \hat{p}(t, x)-\frac{\partial \hat{\sigma}}{\partial \bar{u}}(t, x) \hat{q}(t, x)-\int_{\mathbf{E}} \frac{\partial \hat{\theta}}{\partial \bar{u}}(t, x, e) \hat{\gamma}(t, x, e) \nu(d e)\right)\right. \\
& \quad+<\nabla \mathbf{G}(\hat{u}(t, x)), \beta(t, x)>d x d t]+\mathbf{E}\left[\int_{D}<\hat{p}(T, x), \mathcal{Y}(T, x)>d x\right] .
\end{aligned}
$$

Applying Itô formula to $<\hat{p}(T, x), \mathcal{Y}(T, x)>$ and using the dynamics of the adjoint equations, we finally get

$$
\lim _{y \rightarrow 0} \frac{1}{y}(J(\hat{u}+y \beta)-J(\hat{u}))=\mathbf{E}\left[\int_{0}^{T} \int_{D} \mathbf{E}\left[<\nabla_{u} \hat{H}(t, x), \beta(t, x)>\mid \mathcal{E}_{t}\right] d x d t\right],
$$

where

$$
<\nabla_{u} \hat{H}(t, x), \beta(t, x)>=\frac{\partial H}{\partial u}(t, x) \beta(t, x)+\frac{\partial H}{\partial \bar{u}}(t, x)<\nabla \mathbf{G}(\hat{u}), \beta(t, x)>_{\mathbf{L}^{2}(\mathbf{P})} .
$$

We conclude that

$$
\lim _{y \rightarrow 0} \frac{1}{y}(J(\hat{u}+y \beta)-J(\hat{u}))=0
$$

if and only if

$$
\mathbf{E}\left[\int_{0}^{T} \int_{D} \mathbf{E}\left[<\nabla_{u} \hat{H}(t, x), \beta(t, x)>\mid \mathcal{E}_{t}\right] d x d t\right]=0 .
$$

In particular this holds for all $\beta \in \mathcal{A}_{\mathcal{E}}$ which takes the form

$$
\beta(t, x)=\theta(\omega, x) \chi_{[s, T]}(t) ; t \in[0, T],
$$


for a fixed $s \in[0, T)$, where $\theta(\omega, x)$ is a bounded $\mathcal{E}_{s}$-measurable random variable. We thus get that this is again equivalent to

$$
\mathbf{E}\left[\int_{s}^{T} \int_{D} \mathbf{E}\left[<\nabla_{u} \hat{H}(t, x), \theta>\mid \mathcal{E}_{t}\right] d x d t\right]=0 .
$$

We now differentiate with respect to $s$ and derive that

$$
\mathbf{E}\left[\int_{D} \mathbf{E}\left[<\nabla_{u} \hat{H}(s, x), \theta>\mid \mathcal{E}_{s}\right] d x\right]=0 .
$$

Since this holds for all bounded $\mathcal{E}_{s}$-measurable random variable $\theta$, we can easily conclude that

$$
\lim _{y \rightarrow 0} \frac{1}{y}(J(\hat{u}+y \beta)-J(\hat{u}))=0
$$

is equivalent to

$$
\mathbf{E}\left[\frac{\partial \hat{H}}{\partial u}(t, x) \mid \mathcal{E}_{t}\right]+\mathbf{E}\left[\frac{\partial \hat{H}}{\partial \bar{u}}(t, x)\right] \nabla \mathbf{G}(\hat{u}(t, x))=0 \text { a.s., for all }(t, x) \in[0, T] \times D .
$$

\subsection{Application to the optimal harvesting example}

We now return to the problem of optimal harvesting from a population in a lake $D$ stated in the motivating example. Thus we suppose the density $Y(t, x)$ of the population at time $t \in[0, T]$ and at point $x \in D$ is given by the stochastic reaction-diffusion equation (1.1), and the performance criterion is assumed to be as in (1.4). For simplicity, we choose $d=1$ and $\mathcal{E}_{t}=\mathcal{F}_{t}$. In this case the Hamiltonian gets the following form

$$
H(t, x, y, \bar{y}, u, \bar{u}, p, q, \gamma)=\log (y u)+[b(t, x) \bar{y}-y u] p+\sigma(t, x) y q+\int_{\mathbf{R}^{*}} \theta(t, x, e) y \gamma(e) \nu(d e),
$$

and the adjoint BSDE becomes

$$
\begin{aligned}
& d p(t, x)=\left[-\frac{1}{2} \frac{\partial^{2}}{\partial^{2} x} p(t, x)+\frac{1}{Y(t, x)}+\sigma(t, x) q(t, x)+\int_{\mathbf{R}^{*}} \theta(t, x, e) \gamma(t, x, e) \nu(d e)\right. \\
& \quad-u(t, x) p(t, x)-\mathbf{E}[b(t, x) p(t, x)]] d t+q(t, x) d W_{t}+\int_{\mathbf{R}^{*}} \gamma(t, x, e) \tilde{N}(d t, d e) \\
& p(T, x)=\alpha(x), x \in D, \\
& p(t, x)=0, \quad(t, x) \in(0, T) \times \partial D .
\end{aligned}
$$

We now apply the necessary maximum principle which implies the fact that if $u$ is an optimal control then it satisfies the first order condition

$$
u(t, x)=\frac{1}{Y(t, x) p(t, x)} .
$$

We summarize our results as follows: 
Theorem 2.4 Assume that the conditions of Theorem 2.3 hold. Suppose a harvesting rate process $u(t, x)$ is optimal for the optimization problem (1.5). Then

$$
u(t, x)=\frac{1}{Y(t, x) p(t, x)},
$$

where $p(t, x)$ solves the MFBSPDE

$$
\begin{aligned}
& d p(t, x)=\left[-\frac{1}{2} \frac{\partial^{2}}{\partial^{2} x} p(t, x)+\frac{1}{Y(t, x)}+\sigma(t, x) q(t, x)+\int_{\mathbf{R}^{*}} \theta(t, x, e) \gamma(t, x, e) \nu(d e)-\mathbf{E}[b(t, x) p(t, x)]\right. \\
& -u(t, x) p(t, x)] d t+q(t, x) d W_{t}+\int_{\mathbf{R}^{*}} \gamma(t, x, e) \tilde{N}(d t, d e) \\
& p(T, x)=\alpha(x), x \in D . \\
& p(t, x)=0, \quad(t, x) \in(0, T) \times \partial D .
\end{aligned}
$$

\section{Existence and uniqueness results for general forward mean-field SPDEs with Lévy noise}

We address here the problem of existence and uniqueness of the solution of forward meanfield SPDE (2.6) with general mean-field operator, introduced in Section 2. In order to do this, we first describe the general framework. Let $V, H$ be two separable Hilbert spaces such that $V$ is continously, densely imbedded in $H$. Identifying $H$ with its dual we have

$$
V \subset H \approx H^{*} \subset V^{*}
$$

where we have denoted by $V^{*}$ the topological dual of $V$. Let $L$ be a bounded linear operator from $V$ to $V^{*}$ satisfying the following coercivity hypothesis: There exist constants $\chi>0$ and $\zeta \geq 0$ such that

$$
2<-L u, u>+\chi|u|_{H}^{2} \geq \zeta\|u\|_{V}^{2} \text { for all } u \in V
$$

where $\langle L u, u\rangle=L u(u)$ denotes the action of $L u \in V^{*}$ on $u \in V$ and $|\cdot|_{H}\left(\right.$ resp. $\left.\|\cdot\|_{V}\right)$ the norm associated to the Hilbert space $H$ (resp. $V$ ).

Let us introduce the notation adopted in this section.

- $\mathcal{P}$ is the predictable $\sigma$-algebra on $[0, T] \times \Omega$;

- $\mathbf{L}_{\nu}^{2}(H)$ is the set of measurable functions $k:(\mathbf{E}, \mathcal{B}(\mathbf{E})) \mapsto(H, \mathcal{B}(H))$ such that $\|k\|_{\mathbf{L}_{\nu}^{2}(H)}:=\left(\int_{\mathbf{E}}|k(e)|_{H}^{2} \nu(d e)\right)^{\frac{1}{2}}<\infty ;$

- $\mathbf{L}^{2}(\Omega, H)$ is the set of measurable functions $k:(\Omega, \mathcal{F}) \mapsto(H, \mathcal{B}(H))$ such that $\mathbf{E}\left[|k|_{H}^{2}\right]<$ $\infty$;

- $\mathbf{L}^{2}\left(\Omega, \mathbf{L}_{\nu}^{2}(H)\right)$ is the set of measurable functions $k:(\Omega, \mathcal{F}) \mapsto\left(\mathbf{L}_{\nu}^{2}(H), \mathcal{B}\left(\mathbf{L}_{\nu}^{2}(H)\right)\right)$ such that $\mathbf{E}\left[\|k\|_{\mathbf{L}_{\nu}^{2}(H)}^{2}\right]<\infty$ 
- $\mathbf{L}^{2}(\Omega \times[0, T], H)\left(\right.$ resp. $\left.\mathbf{L}_{2}(\Omega \times[0, T], V)\right)$ is the set of $\mathcal{F}_{t}$-adapted $H$-valued (resp. $V$ valued) processes $\Phi: \Omega \times[0, T] \mapsto H\left(\right.$ resp.V) such that $\|\Phi\|_{\mathbf{L}^{2}(\Omega \times[0, T], H)}^{2}:=\mathbf{E}\left[\int_{0}^{T}|\Phi(t)|_{H}^{2} d t\right]<$ $\infty\left(\right.$ resp. $\left.\|\Phi\|_{\mathbf{L}^{2}(\Omega \times[0, T], V)}^{2}:=\mathbf{E}\left[\int_{0}^{T}\|\Phi(t)\|_{V}^{2} d t\right]<\infty\right)$.

- $\mathbf{L}^{2}(\Omega \times[0, T] \times \mathbf{E}, H)$ is the set of all the $\mathcal{P} \times \mathcal{B}(\mathbf{E})$-measurable $H$-valued maps $\theta$ : $\Omega \times[0, T] \times \mathbf{E} \mapsto H$ satisfying $\|\theta\|_{\mathbf{L}^{2}(\Omega \times[0, T] \times \mathbf{E}, H)}:=\mathbf{E}\left[\int_{0}^{T} \int_{\mathbf{E}}|\Phi(t, e)|_{H}^{2} \nu(d e) d t\right]<\infty$.

- $\mathbf{S}^{2}(\Omega \times[0, T], H)$ denotes the set of $\mathcal{F}_{t}$-adapted $H$-valued cadlag processes $\Phi: \Omega \times$ $[0, T] \mapsto H$ such that $\|\Phi\|_{\mathbf{S}^{2}(\Omega \times[0, T], H)}^{2}:=\mathbf{E}\left[\sup _{0 \leq t \leq T}|\Phi(t)|_{H}^{2}\right]<\infty$.

The mean field SPDE under study is:

$d Y_{t}=\left[L Y_{t}+b\left(t, Y_{t}, \mathbf{F}\left(Y_{t}\right)\right)\right] d t+\sigma\left(t, Y_{t}, \mathbf{F}\left(Y_{t}\right)\right) d W_{t}+\int_{\mathbf{E}} \theta\left(t, Y_{t}, \mathbf{F}\left(Y_{t}\right), e\right) \tilde{N}(d t, d e) ; \quad(t, x) \in(0, T) \times D$.

We recall that this equation should be understood in the weak sense.

Before giving the main result of this section, we make the following assumption on the coefficients $b, \sigma, \theta$ and the operator $\mathbf{F}$ which appear in the above mean-field SPDE.

Assumption 3.1 The maps $b: \Omega \times[0, T] \times H \times H \mapsto H, \sigma: \Omega \times[0, T] \times H \times H \mapsto H$ are $\mathcal{P} \times \mathcal{B}(H) \times \mathcal{B}(H) / \mathcal{B}(H)$-measurable. The map $\theta: \Omega \times[0, T] \times H \times H \times \mathbf{E} \mapsto H$ is $\mathcal{P} \times \mathcal{B}(\mathbf{E}) \times \mathcal{B}(H) \times \mathcal{B}(H) / \mathcal{B}(H)$-measurable. There exist a constant $C<\infty$ such that

$$
\begin{aligned}
& \left|b\left(t, y_{1}, \bar{y}_{1}\right)-b\left(t, y_{2}, \bar{y}_{2}\right)\right|_{H}+\left|\sigma\left(t, y_{1}, \bar{y}_{1}\right)-\sigma\left(t, y_{2}, \bar{y}_{2}\right)\right|_{H}+\int_{\boldsymbol{E}}\left|\theta\left(t, y_{1}, \bar{y}_{1}, e\right)-\theta\left(t, y_{2}, \bar{y}_{2}, e\right)\right|^{2} \nu(d e) \\
& \quad \leq C\left(\left|y_{1}-y_{2}\right|_{H}+\left|\bar{y}_{1}-\bar{y}_{2}\right|_{H}\right) \text { a.s. for all }(\omega, t) \in \Omega \times[0, T] .
\end{aligned}
$$

We also assume that there exists $C<\infty$ such that:

$|b(t, y, \bar{y})|_{H}^{2}+|\sigma(t, y, \bar{y})|_{H}^{2}+\int_{\boldsymbol{E}}|\theta(t, y, \bar{y}, e)|_{H}^{2} \nu(d e) \leq C\left(1+|y|_{H}^{2}+|\bar{y}|_{H}^{2}\right), \forall(\omega, t) \in \Omega \times[0, T], y, \bar{y} \in \boldsymbol{R}$.

Finally, we assume that the operator $\mathbf{F}: \boldsymbol{L}^{2}(\Omega ; H) \mapsto H$ is Fréchet differentiable.

Theorem 3.1 Under Assumption 3.1, there exists a unique H-valued progressively measurable process $\left(Y_{t}\right)_{t \geq 0}$ satisfying the mean-field SPDE:

(i) $Y \in \boldsymbol{L}^{2}(\Omega \times[0, T], V) \cap \boldsymbol{S}^{2}(\Omega \times[0, T], H)$;

(ii) $Y_{t}=h+\int_{0}^{t}\left[L Y_{s}+b\left(s, Y_{s}, \boldsymbol{F}\left(Y_{s}\right)\right)\right] d s+\int_{0}^{t} \sigma\left(s, Y_{s}, \boldsymbol{F}\left(Y_{s}\right)\right) d W_{s}+\int_{0}^{t} \int_{\mathbf{E}} \theta\left(s_{-}, Y_{s_{-}}, \boldsymbol{F}\left(Y_{s_{-}}\right), e\right) \tilde{N}(d t, d e)$;

(iii) $Y_{0}=h \in H$.

\section{Proof. I. Existence of the solution}

Let $Y_{t}^{0}:=h, t \geq 0$. For $n \geq 0$, we define $Y^{n+1} \in \mathbf{L}^{2}([0, T] ; V) \cap \mathbf{S}^{2}([0, T] ; H)$ to be the unique solution to the following equation:

$$
d Y_{t}^{n+1}=L Y_{t}^{n+1} d t+b\left(t, Y_{t}^{n+1}, \mathbf{F}\left(Y_{t}^{n}\right)\right) d t+\sigma\left(t, Y_{t}^{n+1}, \mathbf{F}\left(Y_{t}^{n}\right)\right) d W_{t}+\int_{\mathbf{E}} \theta\left(t_{-}, Y_{t_{-}}^{n+1}, \mathbf{F}\left(Y_{t_{-}}^{n}\right), e\right) \tilde{N}(d t, d e)
$$


The solution $Y^{n+1}$ of this equation follows by Proposition 3.1 in [17]. Let us now show that the sequence $\left\{Y^{n}, n \geq 1\right\}$ is a Cauchy sequence in the spaces $\mathbf{L}^{2}(\Omega \times[0, T], V)$ and $\mathbf{S}^{2}(\Omega \times[0, T], H)$. By applying Itô formula, we get

$$
\begin{aligned}
& \left|Y_{t}^{n+1}-Y_{t}^{n}\right|_{H}^{2}=2 \int_{0}^{t}<Y_{s}^{n+1}-Y_{s}^{n}, L\left(Y_{s}^{n+1}-Y_{s}^{n}\right)>d s \\
& +2 \int_{0}^{t}<Y_{s}^{n+1}-Y_{s}^{n}, b\left(s, Y_{s}^{n}, \mathbf{F}\left(Y_{s}^{n}\right)\right)-b\left(s, Y_{s}^{n-1}, \mathbf{F}\left(Y_{s}^{n-1}\right)\right)>_{H} d s \\
& +2 \int_{0}^{t}<Y_{s}^{n+1}-Y_{s}^{n}, \sigma\left(s, Y_{s}^{n+1}, \mathbf{F}\left(Y_{s}^{n}\right)\right)-\sigma\left(s, Y_{s}^{n}, \mathbf{F}\left(Y_{s}^{n-1}\right)\right)>_{H} d W_{s} \\
& +\int_{0}^{t}\left|\sigma\left(s, Y_{s}^{n}, \mathbf{F}\left(Y_{s}^{n}\right)\right)-\sigma\left(s, Y_{s}^{n-1}, \mathbf{F}\left(Y_{s}^{n-1}\right)\right)\right|_{H}^{2} d s \\
& +\int_{0}^{t} \int_{\mathbf{E}}\left[\left|\theta\left(s, Y_{s-}^{n}, \mathbf{F}\left(Y_{s-}^{n}\right), e\right)-\theta\left(s, Y_{s-}^{n-1}, \mathbf{F}\left(Y_{s-}^{n-1}\right), e\right)\right|_{H}^{2}\right] \tilde{N}(d s, d e) \\
& +2 \int_{0}^{t} \int_{\mathbf{E}}<Y_{s^{-}}^{n+1}-Y_{s^{-}}^{n}, \theta\left(s, Y_{s^{-}}^{n}, \mathbf{F}\left(Y_{s^{-}}^{n}\right), e\right)-\theta\left(s, Y_{s^{-}}^{n-1}, \mathbf{F}\left(Y_{s^{-}}^{n-1}\right), e\right)>_{H} \tilde{N}(d s, d e) \\
& +\int_{0}^{t} \int_{\mathbf{E}}\left[\left|\theta\left(s, Y_{s-}^{n}, \mathbf{F}\left(Y_{s-}^{n}\right), e\right)-\theta\left(s, Y_{s-}^{n-1}, \mathbf{F}\left(Y_{s-}^{n-1}\right), e\right)\right|_{H}^{2} \nu(d e) d s .\right.
\end{aligned}
$$

Using Burkholder-Davis-Gundy and Cauchy-Schwarz inequalities and the coercivity assumption (3.34) on the operator $L$, we obtain that

$$
\begin{aligned}
\mathbf{E} & \left.\sup _{0 \leq s \leq t}\left|Y_{s}^{n+1}-Y_{s}^{n}\right|_{H}^{2}\right] \leq-\chi \mathbf{E}\left[\int_{0}^{t}\left|Y_{s}^{n+1}-Y_{s}^{n}\right|_{V}^{2} d s\right]+C \mathbf{E}\left[\int_{0}^{t}\left[\left|Y_{s}^{n+1}-Y_{s}^{n}\right|_{H}^{2} d s\right]\right. \\
& +\frac{1}{2} \mathbf{E}\left[\sup _{0 \leq s \leq t}\left|Y_{s}^{n+1}-Y_{s}^{n}\right|_{H}^{2} d s\right]+C \mathbf{E}\left[\int_{0}^{t}\left[\left|b\left(s, Y_{s}^{n}, \mathbf{F}\left(Y_{s}^{n}\right)\right)-b\left(s, Y_{s}^{n-1}, \mathbf{F}\left(Y_{s}^{n-1}\right)\right)\right|_{H}^{2} d s\right]\right. \\
& +C \mathbf{E}\left[\int_{0}^{t}\left[\left|\sigma\left(s, Y_{s}^{n}, \mathbf{F}\left(Y_{s}^{n}\right)\right)-\sigma\left(s, Y_{s}^{n-1}, \mathbf{F}\left(Y_{s}^{n-1}\right)\right)\right|_{H}^{2} d s\right]\right. \\
& +C \mathbf{E}\left[\int_{0}^{t} \int_{\mathbf{E}}\left[\left|\theta\left(s, Y_{s}^{n}, \mathbf{F}\left(Y_{s}^{n}\right), e\right)-\theta\left(s, Y_{s}^{n-1}, \mathbf{F}\left(Y_{s}^{n-1}\right), e\right)\right|_{H}^{2} \nu(d e) d s\right] .\right.
\end{aligned}
$$

By the Lipschitz properties of $b, \sigma$ and $\theta$, we deduce

$$
\begin{aligned}
\mathbf{E}\left[\sup _{0 \leq s \leq t}\left|Y_{s}^{n+1}-Y_{s}^{n}\right|_{H}^{2}\right] \leq & C \mathbf{E}\left[\int_{0}^{t}\left[\left|Y_{s}^{n+1}-Y_{s}^{n}\right|_{H}^{2} d s\right]+C \mathbf{E}\left[\int_{0}^{t}\left[\left|Y_{s}^{n}-Y_{s}^{n-1}\right|_{H}^{2} d s\right]\right.\right. \\
& +C \mathbf{E}\left[\int_{0}^{t}\left|\mathbf{F}\left(Y_{s}^{n}\right)-\mathbf{F}\left(Y_{s}^{n-1}\right)\right|_{H}^{2} d s\right] .
\end{aligned}
$$

We use the mean theorem and obtain the existence for each $n \in \mathbb{N}, t \in[0, T]$ of a random variable $\tilde{Y}^{n}(t) \in \mathbf{L}^{2}(\Omega, H)$ such that

$$
\left|\mathbf{F}\left(Y_{t}^{n}\right)-\mathbf{F}\left(Y_{t}^{n-1}\right)\right|_{H} \leq\left\|\nabla \mathbf{F}\left(\tilde{Y}^{n}(t)\right)\right\|\left\|Y_{t}^{n}-Y_{t}^{n-1}\right\|_{\mathbf{L}^{2}(\Omega ; H)} .
$$

The two above relations (3.37) and (3.38) lead to:

$$
\mathbf{E}\left[\sup _{0 \leq s \leq t}\left|Y_{s}^{n+1}-Y_{s}^{n}\right|_{H}^{2}\right] \leq C \mathbf{E}\left[\int_{0}^{t}\left[\left|Y_{s}^{n+1}-Y_{s}^{n}\right|_{H}^{2} d s\right]+C \mathbf{E}\left[\int_{0}^{t}\left|Y_{s}^{n}-Y_{s}^{n-1}\right|_{H} d s\right] .\right.
$$


Let us now define

$$
a_{t}^{n}=\mathbf{E}\left[\sup _{0 \leq s \leq t}\left|Y_{s}^{n}-Y_{s}^{n-1}\right|_{H}^{2} d s\right] \quad A_{t}^{n}=\int_{0}^{t} a_{s}^{n} d s .
$$

Using (3.39), we obtain:

$$
a_{t}^{n+1} \leq C A_{t}^{n+1}+C A_{t}^{n} .
$$

We multiply the above inequality by $e^{-C t}$ and derive

$$
\frac{d\left(A_{t}^{n+1} e^{-C t}\right)}{d t} \leq C e^{-C t} A_{t}^{n}
$$

which allows us to conclude that

$$
A_{t}^{n+1} \leq C e^{C t} \int_{0}^{t} e^{-C s} A_{s}^{n} d s \leq C e^{C t} t A_{t}^{n}
$$

This inequality together with (3.40) gives

$$
a_{t}^{n+1} \leq C^{2} e^{C t} t A_{t}^{n}+C A_{t}^{n} \leq C_{T} \int_{0}^{t} A_{s}^{n} d s,
$$

where $C_{T}$ is a given constant. By iteration for all $n$, we finally obtain

$$
\mathbf{E}\left[\sup _{0 \leq s \leq T}\left|Y_{s}^{n+1}-Y_{s}^{n}\right|_{H}^{2}\right] \leq C \frac{\left(C_{T} T\right)^{n}}{n !} .
$$

This implies that we can find $Y \in \mathbf{S}_{2}(\Omega \times[0, T] ; H)$ such that

$$
\lim _{n \rightarrow \infty} \mathbf{E}\left[\sup _{0 \leq s \leq t}\left|Y_{s}^{n}-Y_{s}\right|_{H}^{2} d s\right]=0
$$

By (3.36), we remark that $Y^{n}$ also converges to $Y$ in $\mathbf{L}^{2}(\Omega \times[0, T], V)$. Passing to the limit in (3.35), we obtain that $Y$ satisfies this equation.

\section{Uniqueness of the solution}

Let $Y_{1}$ and $Y_{2}$ be two solutions in $\mathbf{S}^{2}(\Omega \times[0, T], H) \cap \mathbf{L}^{2}(\Omega \times[0, T], V)$. Applying Itô formula, we have

$$
\begin{aligned}
\left|Y_{t}^{1}-Y_{t}^{2}\right|_{H}^{2}= & -2 \int_{0}^{t}<Y_{s}^{1}-Y_{s}^{2}, L\left(Y_{s}^{1}-Y_{s}^{2}\right)>d s \\
& +2 \int_{0}^{t}<Y_{s}^{1}-Y_{s}^{2}, b\left(s, Y_{s}^{1}, \mathbf{F}\left(Y_{s}^{1}\right)\right)-b\left(s, Y_{s}^{2}, \mathbf{F}\left(Y_{s}^{2}\right)\right)>_{H} d s \\
& +2 \int_{0}^{t}<Y_{s}^{1}-Y_{s}^{2}, \sigma\left(s, Y_{s}^{1}, \mathbf{F}\left(Y_{s}^{1}\right)\right)-\sigma\left(s, Y_{s}^{2}, \mathbf{F}\left(Y_{s}^{2}\right)\right)>_{H} d W_{s} \\
& +\int_{0}^{t}\left|\sigma\left(s, Y_{s}^{1}, \mathbf{F}\left(Y_{s}^{1}\right)\right)-\sigma\left(s, Y_{s}^{2}, \mathbf{F}\left(Y_{s}^{2}\right)\right)\right|_{H}^{2} d s \\
& +\int_{0}^{t} \int_{\mathbf{E}}\left[\left|\theta\left(s, Y_{s^{-}}^{1}, \mathbf{F}\left(Y_{s^{-}}^{1}\right), e\right)-\theta\left(s, Y_{s^{-}}^{2}, \mathbf{F}\left(Y_{s^{-}}^{2}\right), e\right)\right|_{H}^{2}\right. \\
& \left.+2<Y_{s^{-}}^{1}-Y_{s^{-}}^{2}, \theta\left(s, Y_{s^{-}}^{1}, \mathbf{F}\left(Y_{s^{-}}^{1}\right), e\right)-\theta\left(s, Y_{s^{-}}^{2}, \mathbf{F}\left(Y_{s^{-}}^{2}\right), e\right)>\right] \tilde{N}(d s, d e) \\
& +\int_{0}^{t} \int_{\mathbf{E}}\left|\theta\left(s, Y_{s^{-}}^{1}, \mathbf{F}\left(Y_{s^{-}}^{1}\right), e\right)-\theta\left(s, Y_{s^{-}}^{2}, \mathbf{F}\left(Y_{s^{-}}^{2}\right), e\right)\right|_{H}^{2} d s \nu(d e) .
\end{aligned}
$$


Using now the coercivity assumption on the operator $L$, the Lipschitz property of $b, \sigma, \theta$ and the boundness of the Fréchet derivative of the operator $\mathbf{F}$, we finally obtain:

$$
\begin{array}{r}
\mathbf{E}\left[\left|Y_{t}^{1}-Y_{t}^{2}\right|_{H}^{2}\right] \leq-\alpha \mathbf{E}\left[\int_{0}^{t}\left|Y_{s}^{1}-Y_{s}^{2}\right|_{V}^{2} d s\right]+C \mathbf{E}\left[\int_{0}^{t}\left|Y_{s}^{1}-Y_{s}^{2}\right|_{H}^{2} d s\right] \\
+\frac{1}{2} \mathbf{E}\left[\sup _{0 \leq s \leq t}\left|Y_{t}^{1}-Y_{t}^{2}\right|_{H}^{2}\right]+C \mathbf{E}\left[\int_{0}^{t}\left|b\left(s, Y_{s}^{1}, \mathbf{F}\left(Y_{s}^{1}\right)\right)-b\left(s, Y_{s}^{2}, \mathbf{F}\left(Y_{s}^{2}\right)\right)\right|_{H}^{2} d s\right] \\
+C \mathbf{E}\left[\int_{0}^{t}\left|\sigma\left(s, Y_{s}^{1}, \mathbf{F}\left(Y_{s}^{1}\right)\right)-\sigma\left(s, Y_{s}^{2}, \mathbf{F}\left(Y_{s}^{2}\right)\right)\right|_{H}^{2} d s\right]+ \\
C \mathbf{E}\left[\int_{0}^{t} \int_{\mathbf{E}}\left|\theta\left(s, Y_{s}^{1}, \mathbf{F}\left(Y_{s}^{1}\right)\right)-\theta\left(s, Y_{s}^{2}, \mathbf{F}\left(Y_{s}^{2}\right)\right)\right|_{H}^{2} \nu(d e) d s\right] \\
\leq C \mathbf{E}\left[\int_{0}^{t}\left|Y_{s}^{1}-Y_{s}^{2}\right|_{H}^{2} d s\right] .
\end{array}
$$

We thus deduce that $Y_{t}^{1}=Y_{t}^{2}$.

\section{Existence and uniqueness results for general mean- field backward SPDEs with Lévy noise}

In this section we give an existence and uniqueness result for mean-field backward SPDEs with jumps. The analysis will be carried out in a general case, where there exists a general mean-field operator acting on each composant of the solution.

We consider the same framework as in the previous section. Let $A$ be a bounded linear operator from $V$ to $V^{*}$ satisfying the following coercivity hypothesis: There exist constants $\alpha>0$ and $\lambda \geq 0$ such that

$$
2<A u, u>+\lambda|u|_{H}^{2} \geq \alpha\|u\|_{V}^{2} \text { for all } u \in V
$$

where $\langle A u, u\rangle=A u(u)$ denotes the action of $A u \in V^{*}$ on $u \in V$.

Assumption 4.2 Let $f:[0, T] \times \Omega \times H \times H \times H \times H \times \boldsymbol{L}_{\nu}^{2}(H) \times \boldsymbol{L}_{\nu}^{2}(H) \rightarrow H$ be a $\mathcal{P} \times \mathcal{B}(H) \times \mathcal{B}(H) \times \mathcal{B}(H) \times \mathcal{B}(H) \times \mathcal{B}\left(\boldsymbol{L}_{\nu}^{2}(H)\right) \times \mathcal{B}\left(\boldsymbol{L}_{\nu}^{2}(H)\right) / \mathcal{B}(H)$ measurable. There exists a constant $C<\infty$ such that

$$
\begin{aligned}
\mid f\left(t, \omega, y_{1}, \tilde{y}_{1}, z_{1}, \tilde{z}_{1}, q_{1}, \tilde{q}_{1}\right) & -\left.f\left(t, \omega, y_{2}, \tilde{y}_{2}, z_{2}, \tilde{z}_{2}, q_{2}, \tilde{q}_{2}\right)\right|_{H} \leq C\left(\left|y_{1}-y_{2}\right|_{H}+\left|\tilde{y}_{1}-\tilde{y}_{2}\right|_{H}\right. \\
& \left.+\left|z_{1}-z_{2}\right|_{H}+\left|\tilde{z}_{1}-\tilde{z}_{2}\right|_{H}+\left|q_{1}-q_{2}\right|_{L_{\nu}^{2}(H)}+\left|\tilde{q}_{1}-\tilde{q}_{2}\right|_{L_{\nu}^{2}(H)}\right)
\end{aligned}
$$

for all $t, y_{1}, \tilde{y}_{1}, z_{1}, \tilde{z}_{1}, q_{1}, \tilde{q}_{1}, y_{2}, \tilde{y}_{2}, z_{2}, \tilde{z}_{2}, q_{2}, \tilde{q}_{2}$. We also assume the integrability condition

$$
\mathbf{E}\left[\int_{0}^{T}|f(t, 0,0,0,0,0,0)|_{H}^{2} d t\right]<\infty .
$$

We now give our main result of existence and uniqueness. 
Theorem 4.1 Assume Assumption 4.2 holds. Let $\xi \in \boldsymbol{L}^{2}(\Omega ; H)$. Let $\mathcal{H}: \boldsymbol{L}^{2}(\Omega ; H) \mapsto H$, $\mathcal{J}: \boldsymbol{L}^{2}(\Omega ; H) \mapsto H$ and $\mathcal{K}: \boldsymbol{L}^{2}\left(\Omega, \boldsymbol{L}_{\nu}^{2}(H)\right) \mapsto \boldsymbol{L}_{\nu}^{2}(H)$ be Fréchet differentiable operators. There exists a unique $H \times H \times \boldsymbol{L}_{\nu}^{2}(H)$-valued progressively measurable process $\left(Y_{t}, Z_{t}, U_{t}\right)$ such that

$$
\text { (i) } \mathbf{E}\left[\left|Y_{t}\right|_{H}^{2}\right]<\infty, \mathbf{E}\left[\int_{0}^{T}\left|Z_{t}\right|_{H}^{2}\right]<\infty, \mathbf{E}\left[\int_{0}^{T}\left|U_{t}\right|_{L_{\nu}^{2}(H)}^{2} d t\right]<\infty .
$$

(ii) $\xi=Y_{t}+\int_{t}^{T} A Y_{s} d s+\int_{t}^{T} f\left(s, Y_{s}, \mathcal{H}\left(Y_{s}\right), Z_{s}, \mathcal{J}\left(Z_{s}\right), U_{s}, \mathcal{K}\left(U_{s}\right)\right) d s+\int_{t}^{T} Z_{s} d W_{s}+\int_{t}^{T} \int_{E} U_{s}(e) \tilde{N}(d s, d e)$ for all $0 \leq t \leq T$.

The equation (ii) should be understood in the dual space $V^{*}$.

\section{Proof. I. Existence of the solution}

Set $Y_{t}^{0}=0 ; Z_{t}^{0}=0 ; U_{t}^{0}=0$. We denote by $\left(Y_{t}^{n}, Z_{t}^{n}, U_{t}^{n}\right)$ the unique solution of the mean-field backward stochastic equation:

$$
\begin{cases}d Y_{t}^{n} & =A Y_{t}^{n} d t+f\left(t, Y_{t}^{n}, \mathcal{H}\left(Y_{t}^{n-1}\right), Z_{t}^{n}, \mathcal{J}\left(Z_{t}^{n-1}\right), U_{t}^{n}, \mathcal{K}\left(U_{t}^{n-1}\right)\right) d t+Z_{t}^{n} d W_{t}+\int_{\mathbf{E}} U_{t}^{n}(e) \tilde{N}(d t, d e) \\ Y_{T}^{n} & =\xi\end{cases}
$$

The existence and the uniqueness of a solution $\left(Y_{t}^{n}, Z_{t}^{n}, U_{t}^{n}\right)$ of such an equation has been proved in [13]. By applying Itô's formula, we get

$$
\begin{aligned}
0 & =\left|Y_{T}^{n+1}-Y_{T}^{n}\right|_{H}^{2} \\
& =\left|Y_{t}^{n+1}-Y_{t}^{n}\right|_{H}^{2}+2 \int_{t}^{T}<A\left(Y_{s}^{n+1}-Y_{s}^{n}\right), Y_{s}^{n+1}-Y_{s}^{n}>d s \\
& +2 \int_{t}^{T}<f\left(s, Y_{s}^{n+1}, \mathcal{H}\left(Y_{s}^{n}\right), Z_{s}^{n+1}, \mathcal{J}\left(Z_{s}^{n}\right), U_{s}^{n+1}, \mathcal{K}\left(U_{s}^{n}\right)\right) \\
& \quad-f\left(s, Y_{s}^{n}, \mathcal{H}\left(Y_{s}^{n-1}\right), Z_{s}^{n}, \mathcal{J}\left(Z_{s}^{n-1}\right), U_{s}^{n}, \mathcal{K}\left(U_{s}^{n-1}\right)\right), Y_{s}^{n+1}-Y_{s}^{n}>_{H} d s \\
& +\int_{t}^{T} \int_{\mathbf{E}}\left[\left|Y_{s^{-}}^{n+1}-Y_{s^{-}}^{n}+U_{s}^{n+1}-U_{s}^{n}\right|_{H}^{2}-\left|Y_{s^{-}}^{n+1}-Y_{s^{-}}^{n}\right|_{H}^{2}\right] \tilde{N}(d s, d e)+\int_{t}^{T} \int_{\mathbf{E}}\left[\left|U_{s}^{n+1}(e)-U_{s}^{n}(e)\right|_{H}^{2}\right] \nu(d e \\
& +2 \int_{t}^{T}<Y_{s}^{n+1}-Y_{s}^{n}, d\left(\mathcal{Z}_{s}^{n+1}-\mathcal{Z}_{s}^{n}\right)>_{H}+\int_{t}^{T}\left|Z_{s}^{n+1}-Z_{s}^{n}\right|_{H}^{2} d s,
\end{aligned}
$$

where $\mathcal{Z}_{t}^{n}:=\int_{0}^{t} Z_{s}^{n} d W_{s}$.

We thus get, by taking the expectation and using the coercivity assumption on the 
operator $A$

$$
\begin{aligned}
& \mathbf{E}\left[\left|Y_{t}^{n+1}-Y_{t}^{n}\right|_{H}^{2}\right]+\mathbf{E}\left[\int_{t}^{T}\left|Z_{s}^{n+1}-Z_{s}^{n}\right|_{H}^{2} d s\right]+\mathbf{E}\left[\int_{t}^{T} \int_{\mathbf{E}}\left|U_{s}^{n+1}-U_{s}^{n}\right|_{H}^{2} \nu(d e) d s\right]= \\
& -2 \mathbf{E}\left[<A\left(Y_{s}^{n+1}-Y_{s}^{n}\right), Y_{s}^{n+1}-Y_{s}^{n}>d s\right] \\
& -2 \mathbf{E}\left[\int_{t}^{T}<f\left(s, Y_{s}^{n+1}, \mathcal{H}\left(Y_{s}^{n}\right), Z_{s}^{n+1}, \mathcal{J}\left(Z_{s}^{n}\right), U_{s}^{n+1}, \mathcal{K}\left(U_{s}^{n}\right)\right)\right. \\
& \left.\quad-f\left(s, Y_{s}^{n}, \mathcal{H}\left(Y_{s}^{n-1}\right), Z_{s}^{n}, \mathcal{J}\left(Z_{s}^{n-1}\right), U_{s}^{n}, \mathcal{K}\left(U_{s}^{n-1}\right)\right), Y_{s}^{n+1}-Y_{s}^{n}>d s\right] \leq \\
& \leq \lambda \mathbf{E}\left[\int_{t}^{T}\left|Y_{s}^{n+1}-Y_{s}^{n}\right|_{H}^{2} d s\right]-\alpha \mathbf{E}\left[\int_{t}^{T}\left|Y_{s}^{n+1}-Y_{s}^{n}\right|_{V}^{2} d s\right]- \\
& -2 \mathbf{E}\left[\int_{t}^{T} \quad<f\left(s, Y_{s}^{n+1}, \mathcal{H}\left(Y_{s}^{n}\right), Z_{s}^{n+1}, \mathcal{J}\left(Z_{s}^{n}\right), U_{s}^{n+1}, \mathcal{K}\left(U_{s}^{n}\right)\right)\right. \\
& \left.\quad-f\left(s, Y_{s}^{n}, \mathcal{H}\left(Y_{s}^{n-1}\right), Z_{s}^{n}, \mathcal{J}\left(Z_{s}^{n-1}\right), U_{s}^{n}, \mathcal{K}\left(U_{s}^{n-1}\right)\right), Y_{s}^{n+1}-Y_{s}^{n}>_{H} d s\right] .
\end{aligned}
$$

By using the Cauchy Schwarz inequality and the Lipschitz property of the generator $f$, for each $(t, \omega) \in[0, T] \times \Omega$, we obtain:

$$
\begin{aligned}
& <f\left(s, Y_{s}^{n+1}, \mathcal{H}\left(Y_{s}^{n-1}\right), Z_{s}^{n+1}, \mathcal{J}\left(Z_{s}^{n-1}\right), U_{s}^{n+1}, \mathcal{K}\left(U_{s}^{n-1}\right)\right) \\
& -f\left(s, Y_{s}^{n}, \mathcal{H}\left(Y_{s}^{n-1}\right), Z_{s}^{n}, \mathcal{J}\left(Z_{s}^{n-1}\right), U_{s}^{n}, \mathcal{K}\left(U_{s}^{n-1}\right)\right), Y_{s}^{n+1}-Y_{s}^{n}>_{H} \\
& \leq \mid f\left(s, Y_{s}^{n+1}, \mathcal{H}\left(Y_{s}^{n-1}\right), Z_{s}^{n+1}, \mathcal{J}\left(Z_{s}^{n-1}\right), U_{s}^{n+1}, \mathcal{K}\left(U_{s}^{n-1}\right)\right)-f\left(s, Y_{s}^{n}, \mathcal{H}\left(Y_{s}^{n-1}\right), Z_{s}^{n}, \mathcal{J}\left(Z_{s}^{n-1}\right), U_{s}^{n}, \mathcal{K}\left(U_{s}^{n-1}\right)\right. \\
& \cdot\left|Y_{s}^{n+1}-Y_{s}^{n}\right|_{H} \\
& \leq C\left(\left|\mathcal{H}\left(Y_{s}^{n}\right)-\mathcal{H}\left(Y_{s}^{n-1}\right)\right|_{H}+\left|\mathcal{J}\left(Z_{s}^{n}\right)-\mathcal{J}\left(Z_{s}^{n-1}\right)\right|_{H}+\left|\mathcal{K}\left(U_{s}^{n}\right)-\mathcal{K}\left(U_{s}^{n-1}\right)\right|_{\mathbf{L}_{\nu}^{2}(H)}\right)\left|Y_{s}^{n+1}-Y_{s}^{n}\right|_{H} \\
& +C\left(\left|Y_{s}^{n+1}-Y_{s}^{n}\right|_{H}+\left|Z_{s}^{n+1}-Z_{s}^{n}\right|_{H}+\left|U_{s}^{n+1}-U_{s}^{n}\right|_{\mathbf{L}_{\nu}^{2}(H)}\right)\left|Y_{s}^{n+1}-Y_{s}^{n}\right|_{H} \text {. } \\
& \quad \text { We now appeal to the mean theorem in Hilbert spaces and obtain the existence for } \\
& \text { each } t \in[0, T] \text { of some random variables } \tilde{Y}^{n}(t) \in \mathbf{L}^{2}(\Omega, H), \tilde{Z}^{n}(t) \in \mathbf{L}^{2}(\Omega, H), \tilde{U}^{n}(t) \in \\
& \mathbf{L}^{2}\left(\Omega, \mathbf{L}_{\nu}^{2}(H)\right) \text { such that } \\
& \qquad\left|\mathcal{H}\left(Y_{t}^{n}\right)-\mathcal{H}\left(Y_{t}^{n-1}\right)\right|_{H} \leq\left\|\nabla \mathcal{H}\left(\tilde{Y}^{n}(t)\right)\right\|\left\|Y_{t}^{n}-Y_{t}^{n-1}\right\|_{\mathbf{L}^{2}(\Omega, H)} \\
& \qquad\left|\mathcal{J}\left(Z_{t}^{n}\right)-\mathcal{J}\left(Z_{t}^{n-1}\right)\right|_{H} \leq\left\|\nabla \mathcal{J}\left(\tilde{Z}^{n}(t)\right)\right\|\left\|Z_{t}^{n}-Z_{t}^{n-1}\right\|_{\mathbf{L}^{2}(\Omega, H)} \\
& \qquad\left|\mathcal{K}\left(U_{t}^{n}\right)-\mathcal{K}\left(U_{t}^{n-1}\right)\right|_{H} \leq\left\|\nabla \mathcal{K}\left(\tilde{U}^{n}(t)\right)\right\|\left\|U_{t}^{n}-U_{t}^{n-1}\right\|_{\mathbf{L}^{2}\left(\Omega, \mathbf{L}_{\nu}^{2}(H)\right)}
\end{aligned}
$$

Using (4.42), (4.43), (4.44) together with the boundness of the Fréchet derivatives of the operators $\mathcal{H}, \mathcal{J}, \mathcal{K}$ and the inequality $2 a b \leq \varepsilon a^{2}+\frac{1}{\varepsilon} b^{2}$, we obtain:

$$
\begin{aligned}
& \mathbf{E}\left[\left|Y_{t}^{n+1}-Y_{t}^{n}\right|_{H}^{2}\right]+\mathbf{E}\left[\int_{t}^{T}\left|Z_{s}^{n+1}-Z_{s}^{n}\right|_{H}^{2} d s\right]+\mathbf{E}\left[\int_{t}^{T} \int_{\mathbf{E}}\left|U_{s}^{n+1}(e)-U_{s}^{n}(e)\right|_{H}^{2} \nu(d e) d s\right] \leq \\
& \leq \lambda \mathbf{E}\left[\int_{t}^{T}\left|Y_{s}^{n+1}-Y_{s}^{n}\right|_{H}^{2} d s\right]-\alpha \mathbf{E}\left[\int_{t}^{T}\left|Y_{s}^{n+1}-Y_{s}^{n}\right|_{V}^{2} d s\right]- \\
& +C \varepsilon \mathbf{E}\left[\int_{t}^{T}\left(\left|Y_{s}^{n}-Y_{s}^{n-1}\right|_{H}^{2}+\left|Z_{s}^{n}-Z_{s}^{n-1}\right|_{H}^{2}+\left|U_{s}^{n}-U_{s}^{n-1}\right|_{\mathbf{L}_{\nu}^{2}(H)}^{2}\right) d s\right]+\frac{1}{\varepsilon} \mathbf{E}\left[\int_{t}^{T}\left|Y_{s}^{n+1}-Y_{s}^{n}\right|_{H}^{2} d s\right] \\
& +C \beta \mathbf{E}\left[\int_{t}^{T}\left(\left|Y_{s}^{n+1}-Y_{s}^{n}\right|_{H}^{2}+\left|Z_{s}^{n+1}-Z_{s}^{n}\right|_{H}^{2}+\left|U_{s}^{n+1}-U_{s}^{n}\right|_{\mathbf{L}_{\nu}^{2}(H)}^{2}\right) d s\right]+\frac{1}{\beta} \mathbf{E}\left[\int_{t}^{T}\left|Y_{s}^{n+1}-Y_{s}^{n}\right|_{H}^{2} d s\right],
\end{aligned}
$$


where $C$ is a constant dependind on the Lipschitz constant of $f$ and the bounding constants of the Fréchet derivative operators of $\mathcal{H}, \mathcal{J}, \mathcal{K}$.

Let us choose $\varepsilon \leq \frac{1}{4 C}$ and $\beta \leq \frac{1}{2 C}$. We set $\gamma:=\lambda+C \beta+\frac{1}{\varepsilon}+\frac{1}{\beta}+\frac{1}{2}$ and then multiply the previous inequality by $e^{\gamma t}$. We thus get

$$
\begin{aligned}
& -\frac{d}{d t}\left(e^{\gamma t} \mathbf{E}\left[\int_{t}^{T}\left|Y_{s}^{n+1}-Y_{s}^{n}\right|_{H}^{2} d s\right]\right)+\frac{1}{2} e^{\gamma t} \mathbf{E}\left[\int_{t}^{T}\left|Z_{s}^{n+1}-Z_{s}^{n}\right|_{H}^{2} d s\right] \\
& +\frac{1}{2} \mathbf{E}\left[\int_{t}^{T}\left|Y_{s}^{n+1}-Y_{s}^{n}\right|_{H}^{2} d s\right] e^{\gamma t}+\frac{1}{2} e^{\gamma t} \mathbf{E}\left[\int_{t}^{T}\left|U_{s}^{n+1}-U_{s}^{n}\right|_{\mathbf{L}_{\nu}^{2}(H)}^{2} d s\right]+\alpha e^{\gamma t} \mathbf{E}\left[\int_{t}^{T}\left|Y_{s}^{n+1}-Y_{s}^{n}\right|_{V}^{2} d s\right] \\
& \leq \frac{1}{4} \mathbf{E}\left[\int_{t}^{T}\left|Y_{s}^{n}-Y_{s}^{n-1}\right|_{H}^{2}\right] e^{\gamma t}+\frac{1}{4} \mathbf{E}\left[\int_{t}^{T}\left|Z_{s}^{n}-Z_{s}^{n-1}\right|_{H}^{2}\right] e^{\gamma t}+\frac{1}{4} \mathbf{E}\left[\int_{t}^{T}\left|U_{s}^{n}-U_{s}^{n-1}\right|_{\mathbf{L}_{\nu}^{2}(H)}^{2} d s\right] e^{\gamma t} .
\end{aligned}
$$

We now integrate between 0 and $T$ and obtain:

$$
\begin{aligned}
\mathbf{E}\left[\int_{0}^{T}\left|Y_{s}^{n+1}-Y_{s}^{n}\right|_{H}^{2} d s\right] & +\frac{1}{2} \int_{0}^{T} \mathbf{E}\left[\int_{t}^{T}\left|Y_{s}^{n+1}-Y_{s}^{n}\right|_{H}^{2} d s\right] e^{\gamma t} d t+\frac{1}{2} \int_{0}^{T} \mathbf{E}\left[\int_{t}^{T}\left|Z_{s}^{n+1}-Z_{s}^{n}\right|_{H}^{2} d s\right] e^{\gamma t} d t \\
& +\frac{1}{2} \int_{0}^{T} e^{\gamma t} \mathbf{E}\left[\int_{t}^{T}\left|U_{s}^{n+1}-U_{s}^{n}\right|_{\mathbf{L}_{\nu}^{2}(H)}^{2} d s\right]+\int_{0}^{T} \alpha \mathbf{E}\left[\int_{t}^{T}\left|Y_{s}^{n+1}-Y_{s}^{n}\right|_{V}^{2} d s\right] e^{\gamma t} d t \\
\leq & \frac{1}{4} \int_{0}^{T} \mathbf{E}\left[\int_{t}^{T}\left|Y_{s}^{n}-Y_{s}^{n-1}\right|_{H}^{2} d s\right] e^{\gamma t} d t+\frac{1}{4} \int_{0}^{T} \mathbf{E}\left[\int_{t}^{T}\left|Z_{s}^{n}-Z_{s}^{n-1}\right|_{H}^{2} d s\right] e^{\gamma t} d t \\
& +\frac{1}{4} \int_{0}^{T} \mathbf{E}\left[\int_{t}^{T}\left|U_{s}^{n}-U_{s}^{n-1}\right|_{\mathbf{L}_{\nu}^{2}(H)}^{2} d s\right] e^{\gamma t} d t .
\end{aligned}
$$

From the above inequality it follows that

$$
\begin{aligned}
\int_{0}^{T} \mathbf{E}\left[\int_{t}^{T}\left|Y_{s}^{n}-Y_{s}^{n-1}\right|_{H}^{2}\right] e^{\gamma t} d t & +\int_{0}^{T} \mathbf{E}\left[\int_{t}^{T}\left|Z_{s}^{n}-Z_{s}^{n-1}\right|_{H}^{2}\right] e^{\gamma t} d t+\int_{0}^{T} \mathbf{E}\left[\int_{t}^{T}\left|U_{s}^{n}-U_{s}^{n-1}\right|_{\mathbf{L}_{\nu}^{2}(H)} d s\right] e^{\gamma t} d t \\
& \leq \frac{1}{2^{n}} C .
\end{aligned}
$$

From (4.46) one can deduce

$$
\mathbf{E}\left[\int_{0}^{T}\left|Y_{s}^{n+1}-Y_{s}^{n}\right|_{H}^{2} d s\right] \leq \frac{1}{2^{n}} C .
$$

We now appeal to (4.45) and derive

$$
\begin{aligned}
& \frac{1}{2} \mathbf{E}\left[\int_{0}^{T}\left|Y_{s}^{n+1}-Y_{s}^{n}\right|_{H}^{2} d s\right]+\frac{1}{2} \int_{0}^{T} \mathbf{E}\left[\int_{0}^{T}\left|Z_{s}^{n+1}-Z_{s}^{n}\right|_{H}^{2} d s\right]+\frac{1}{2} \int_{0}^{T} \mathbf{E}\left[\int_{0}^{T}\left|U_{s}^{n}-U_{s}^{n-1}\right|_{\mathbf{L}_{\nu}^{2}(H)}^{2} d s\right] \\
& \leq \gamma \frac{1}{2^{n}} C+\frac{1}{4} \mathbf{E}\left[\int_{0}^{T}\left|Y_{s}^{n}-Y_{s}^{n-1}\right|_{H}^{2} d s\right]+\frac{1}{4} \mathbf{E}\left[\int_{0}^{T}\left|Z_{s}^{n}-Z_{s}^{n-1}\right|_{H}^{2} d s\right]+\frac{1}{4} \mathbf{E}\left[\int_{0}^{T}\left|U_{s}^{n+1}-U_{s}^{n}\right|_{\mathbf{L}_{\nu}^{2}(H)}^{2} d s\right],
\end{aligned}
$$

which implies that

$$
\mathbf{E}\left[\int_{0}^{T}\left|Y_{s}^{n+1}-Y_{s}^{n}\right|_{H}^{2} d s\right]+\int_{0}^{T} \mathbf{E}\left[\int_{0}^{T}\left|Z_{s}^{n+1}-Z_{s}^{n}\right|_{H}^{2} d s\right]+\int_{0}^{T} \mathbf{E}\left[\int_{0}^{T}\left|U_{s}^{n}-U_{s}^{n-1}\right|_{\mathbf{L}_{\nu}^{2}(H)}^{2} d s\right] \leq \frac{1}{2^{n-1}} C \gamma n .
$$


This leads to

$$
\mathbf{E}\left[\int_{0}^{T}\left|Y_{s}^{n+1}-Y_{s}^{n}\right|_{V}^{2} d s\right] \leq\left(\frac{1}{2}\right)^{n-1}(n+1) C \gamma .
$$

Hence, we can conclude that the sequence $\left(Y^{n}, Z^{n}, U^{n}\right), n \geq 1$ is a Cauchy sequence in the Banach space $L^{2}(\Omega \times[0, T], V) \times L^{2}(\Omega \times[0, T], H) \times L^{2}\left(\Omega \times[0, T], L^{2}(\nu)\right)$, and thus converges in the corresponding spaces to $(Y, Z, U)$. The limit $(Y, Z, U)$ satisfies:

$$
Y_{t}+\int_{t}^{T} A Y_{s} d s+\int_{t}^{T} f\left(s, Y_{s}, \mathcal{H}\left(Y_{s}\right), Z_{s}, \mathcal{J}\left(Z_{s}\right), U_{s}, \mathcal{K}\left(U_{s}\right)\right) d s+\int_{t}^{T} Z_{s} d W_{s}+\int_{t}^{T} \int_{\mathbf{E}} U_{s} \tilde{N}(d s, d e)=\xi \text { a.s. }
$$

\section{Uniqueness of the solution}

The proof of the uniqueness of the solution is classical, but we give it for convenience of the reader. Suppose $\left(Y_{t}, Z_{t}, U_{t}\right)$ and $\left(\tilde{Y}_{t}, \tilde{Z}_{t}, \tilde{U}_{t}\right)$ are two solutions. By applying Itô formula, we obtain

$$
\begin{aligned}
& \left.\mathbf{E}\left[\left|Y_{t}-\tilde{Y}_{t}\right|_{H}^{2}\right]+\mathbf{E}\left[\int_{t}^{T}\left|Z_{s}-\tilde{Z}_{s}\right|_{H}^{2} d s\right]+\mathbf{E}\left[\int_{t}^{T}\left|U_{s}-\tilde{U}_{s}\right|_{\mathbf{L}_{\nu}^{2}(H)}^{2}\right] d s\right]= \\
& -\mathbf{E}\left[<A\left(Y_{s}-\tilde{Y}_{s}\right), Y_{s}-\tilde{Y}_{s}>d s\right] \\
& -2 \mathbf{E}\left[\int_{t}^{T}<f\left(s, Y_{s}, \mathcal{H}\left(Y_{s}\right), Z_{s}, \mathcal{J}\left(Z_{s}\right), U_{s}, \mathcal{K}\left(U_{s}\right)\right)\right. \\
& \left.\quad \quad-f\left(s, \tilde{Y}_{s}, \mathcal{H}\left(\tilde{Y}_{s}\right), \tilde{Z}_{s}, \mathcal{J}\left(\tilde{Z}_{s}\right), \tilde{U}_{s}, \mathcal{K}\left(\tilde{U}_{s}\right)\right), \tilde{Y}_{s}-\tilde{Y}_{s}>_{H} d s\right] \\
& \quad \lambda \mathbf{E}\left[\int_{t}^{T}\left|Y_{s}-\tilde{Y}_{s}\right|_{H}^{2} d s\right]-\alpha \mathbf{E}\left[\int_{t}^{T}\left|Y_{s}-\tilde{Y}_{s}\right|_{V}^{2} d s\right]+K \mathbf{E}\left[\int_{t}^{T}\left|Y_{s}-\tilde{Y}_{s}\right|_{H}^{2} d s\right] \\
& +\frac{1}{2} \mathbf{E}\left[\int_{t}^{T}\left|Z_{s}-\tilde{Z}_{s}\right|_{H}^{2} d s\right]+\frac{1}{2} \mathbf{E}\left[\int_{t}^{T}\left|U_{s}-\tilde{U}_{s}\right|_{\mathbf{L}_{\nu}^{2}(H)}^{2} d s\right] .
\end{aligned}
$$

We thus derive that

$$
\mathbf{E}\left[\left|Y_{t}-\tilde{Y}_{t}\right|_{H}^{2}\right] \leq(\lambda+K) \mathbf{E}\left[\int_{t}^{T}\left|Y_{s}-\tilde{Y}_{s}\right|_{H}^{2}\right]
$$

Hence, by Gronwall lemma, we get

$$
Y_{t}=\tilde{Y}_{t}
$$

This also implies that $Z_{t}=\tilde{Z}_{t}$ and $U_{t}=\tilde{U}_{t}$.

\section{A Some results on Banach theory}

We recall here some basic concepts and results from Banach space theory. Let $V$ be an open subset of a Banach space $\mathcal{X}$ with norm $\|\cdot\|$ and let $F: V \mapsto \mathbb{R}$.

(i) We say that $F$ has a directional derivative (or Gâteaux derivative) at $x \in \mathcal{X}$ in the direction $y \in \mathcal{X}$ if

$$
D_{y} F(x):=\lim _{\varepsilon \rightarrow 0} \frac{1}{\varepsilon}(F(x+\varepsilon y)-F(x))
$$


exists.

(ii) We say that $F$ is Fréchet differentiable at $x \in V$ if there exists a linear map $L: \mathcal{X} \mapsto \mathbb{R}$ such that

$$
\lim _{h \rightarrow 0 ; h \in \mathcal{X}} \frac{1}{\|h\|}|F(x+h)-F(x)-L(h)|=0 .
$$

In this case we call $L$ the gradient (or Fréchet derivative) of $F$ at $x$ and we write

$$
L=\nabla F \text {. }
$$

(iii) If $F$ is Fréchet differentiable, then $F$ has a directional derivative in all directions $y \in \mathcal{X}$ and

$$
D_{y} F(x)=\nabla_{x} F(y)=:<\nabla_{x} F, y>.
$$

In particular, if $\mathcal{X}=\mathbf{L}^{2}(P)$ the Fréchet derivative of $F$ at $X \in \mathbf{L}^{2}(P)$, denoted by $\nabla F(X)$, is a bounded linear functional on $\mathbf{L}^{2}(P)$, which we can identify by Riesz theorem with a random variable in $\mathbf{L}^{2}(P)$. For example, if $F(X)=\mathbf{E}[\phi(X)] ; X \in \mathbf{L}^{2}(P)$, where $\phi$ is a real $C^{1}$-function such that $\phi(X) \in \mathbf{L}^{2}(P)$ and $\frac{\partial \phi}{\partial x}(X) \in L^{2}(P)$, then $\nabla F(X)=\frac{\partial \phi}{\partial x}(X)$ and $\nabla F(X)(Y)=\left\langle\frac{\partial \phi}{\partial x}(X), Y\right\rangle_{\mathbf{L}^{2}(P)}=\mathbf{E}\left[\frac{\partial \phi}{\partial x}(X) Y\right]$, for $Y \in \mathbf{L}^{2}(P)$.

\section{References}

[1] D. Andersson, B. Djehiche, A Maximum Principle for SDEs of Mean-Field Type, Applied Mathematics and Optimization, Vol. 63, Issue 3, pp. 341-356, 2011.

[2] A. Bensoussan, Maximum principle and dynamic programming approaches of the optimal control of partially observed diffusions, Stochastics 9, 169-222, 1983.

[3] A.Bensoussan, Stochastic maximum principles for systems with partial information and application to the separation principle. In M.Davis and R. Elliott (editors): Applied Stochastic Analysis. Gordon and Breach 1991, pp. 157-172.

[4] A. Bensoussan, B. Djehiche, H. Tembine and P.Yam, Risk-Sensitive Mean-Field Type Control, preprint, 2017.

[5] R. Buckdahn, B. Djehiche, J.Li, S. Peng, Mean-field backward stochastic differential equations. A limit approach, Annals of Probability 37(4), 1524-1565, 2009.

[6] R.Buckdahn, J.Li, S.Peng, Mean-field Backward Stochastic Differential Equations and Related Partial Differential Equations, Stochastic Processes and their Applications, Volume 119, Issue 10, Pages 3133-3154, 2009.

[7] R.Buckdahn, J.Li, S.Peng, C. Rainer, Mean-field Stochastic Differential Equations and Associated PDEs, The Annals of Probability, Volume 45, Issue 2, Pages 824-878, 2009. 
[8] R. Carmona, F. Delarue, Mean field forward-backward stochastic differential equations, Electron. Commun. Probab., Vol.18, paper no. 68, 2013.

[9] N. El Karoui, S. Peng and M.C. Quenez, Backward stochastic differential equations in finance, Mathematical finance 7, 1-71, 1997.

[10] B. Øksendal, A. Sulem, Applied stochastic control with jumps processes, SpringerVerlag, 2004.

[11] N.C.Framstad, B. Øksendal and A. Sulem, Sufficient stochastic maximum principle for the optimal control of jump diffusions and applications to finance, J. Optim. Theory and Appl. 121, 77-98, 2004.

[12] B. Øksendal, Optimal control of stochastic partial differential equations, Stoch. Anal. and Appl.

[13] Øksendal B, Proske F, Zhang T, Backward stochastic partial differential equations with jumps and application to optimal control of random jump fields, Stochastics $77(5): 381399,2005$.

[14] Øksendal B, Sulem A., Singular stochastic control and optimal stopping with partial information of jump diffusions, SIAM J. Control Optim. 50(4):22542287, 2012.

[15] Øksendal B, Sulem A, Zhang T., Singular stochastic control and optimal stopping of SPDEs, and Backward SPDEs with reflection, Mathematics of Operations Research, http://dx.doi.org/10.1287/moor.2013.0602, 2013.

[16] Pardoux E., Stochastic partial differential equations and filtering of diffusion processes, Stochastics 3:127167, 1979.

[17] G.D.Prato and J. Zabczyk, Stochastic equations in infinite dimensions, Cambridge University Press, 1992.

[18] Prévot CI, Rockner M., A concise cours on stochastic partial differential equations, Lecture notes in Mathematics 1905 (Springer-Verlag, Berlin, Heidelberg), 2007.

[19] Rockner M., Tusheng Z., Stochastic Evolution Equations of Jump Type: Existence, Uniqueness and Large Deviation Principles, Potential Anal, 26: 255. doi:10.1007/s11118-006-9035-z, 2007.

[20] R. Situ, On solutions of backward stochastic differential equations with jumps and applications, Stochastic Processes and their Applications 66, 209-236, 1997.

[21] M.Tang and Q.Meng, Forward and Backward Mean-Field Stochastic Partial Differential Equation and Optimal Control, preprint 2016 https://arxiv.org/pdf/1610.02486.pdf. 